\title{
CARTOGRAPHIE DE LA DÉFORESTATION DANS LE DÉPARTEMENT DE L'ALIBORI (NORD DU BÉNIN) GRÂCE AUX IMAGES SATELLITAIRES SPOT
}

\author{
Mama DJAOUGA ${ }^{1}$, Ousséni AROUNA ${ }^{1 \& 2}$, Soufouyane ZAKARI ${ }^{1}$, Sébastien KOUTA ${ }^{1}$, \\ Yaya ISSIFOU MOUMOUNI ${ }^{1}$, Benoit MERTENS ${ }^{3}$, Ismaïla TOKO IMOROU ${ }^{1}$ \& Omer THOMAS ${ }^{1}$
}

1. Laboratoire de cartographie (LaCarto), Institut de géographie, de l'aménagement du territoire et de l'environnement (IGATE), université d'Abomey-Calavi (UAC). 10 BP 1082 Cotonou-Houéyiho, Bénin

2. Laboratoire de géosciences, de l'environnement et applications, Ecole nationale supérieure des travaux publics, université nationale des sciences, technologies, ingénierie et mathématiques (UNSTIM), Benin

3. Institut de recherche pour le développement (IRD)

Auteur correspondant : Mama DJAOUGA, maloud75@gmail.com

\begin{abstract}
Résumé
Dans le département de l'Alibori, la production cotonnière, l'élevage bovin, essentiellement extensif et transhumant, l'exploitation forestière, les influences climatiques sahéliennes et l'urbanisation sont autant de facteurs qui accélèrent la déforestation. L'objectif de la présente recherche est d'évaluer le niveau de déforestation dans le département de l'Alibori à partir de l'imagerie satellitaire. Les données planimétriques issues des images SPOT 5 et 7 de 2005 et de 2015, fournies par le programme OSFACO, ont été interprétées et analysées par les auteurs. Le traitement numérique des images satellitaires a été réalisé à l'aide du logiciel QGIS 2.18.2, et notamment grâce au module Train Random Forest Image Classifier contenu dans la boîte à outils Orfeo. Le contrôle-terrain a été effectué à partir de 852 points GPS. La précision moyenne des cartes d'occupation des terres est d'environ $96 \%$. Les résultats révèlent une régression des formations végétales naturelles au profit des formations anthropiques. Les forêts galeries, les forêts denses sèches, les forêts claires et savanes boisées et les savanes arborées et arbustives ont été converties en mosaïques de champs et de jachères ; et en habitat humain. Les terroirs villageois sont plus touchés par la déforestation que les aires protégées qui occupent près de $45 \%$ de la superficie du département. Le taux de déforestation sur l'ensemble du département est de 1,83\%. Ce taux est de 3,10\% dans les terroirs villageois et de $0,70 \%$ dans les aires protégées. Ainsi, $14,96 \%$ de la superficie totale du département, dont $20,45 \%$ des terroirs villageois et seulement $7,40 \%$ des aires protégées, sont touchés par la déforestation. Par ailleurs, 2,66 \% du département est touché par la dégradation des terres forestières ; $1,40 \%$ de cette entité ont été reconstitués naturellement et seulement $0,35 \%$ ont connu une amélioration. La restauration de ces zones dégradées s'avère nécessaire dans le département de l'Alibori, qui constitue le bassin cotonnier du Bénin.
\end{abstract}

Mots-clés : déforestation, images satellitaires SPOT, aires protégées, dégradation, département de l'Alibori, Bénin

\begin{abstract}
In the department of Alibori, cotton production, essentially extensive and transhumant cattle breeding, logging, Sahelian climatic influences, and urbanization are all factors that accelerate deforestation. The objective of this research is to assess deforestation in the department of Alibori using satellite imagery. Planimetric data from the 2005 and 2015 SPOT 5 and 7 images, provided by the spatial observation of tropical forests (OSFACO) project, were interpreted and analyzed. Digital processing of the satellite images was carried out using QGIS 2.18.2 software, in particular the Train Random Forest Image Classifier module contained in the Orfeo ToolBox. Ground control was carried out through 852 GPS points. The average accuracy of the land cover maps was approximately $96 \%$. The results revealed a decline in natural vegetation types in favor of mosaics of fields and fallow land. Gallery forests, dry forests, woodlands, and wooded and shrubby savannas were converted into mosaics of fields and fallow land, as well as settlements. Surrounding villages were more affected by deforestation than protected areas, which occupy nearly $45 \%$ of the department's surface area. The rate of deforestation throughout the department was $1.83 \%$. The rate was $3.10 \%$ in village areas and $0.70 \%$ in protected areas. Thus, $14.96 \%$ of the department's total surface area was affected by deforestation, including more than $20.45 \%$ of village areas and only $7.40 \%$ of protected areas. In addition, $2.66 \%$ of the department was affected by degradation; $1.40 \%$ was naturally restored, and only $0.35 \%$ was improved. Restoring these degraded areas is necessary in the department of Alibori, which constitutes Benin's cotton belt.
\end{abstract}

Keywords: deforestation, SPOT satellite images, protected areas, degradation, department of Alibori, Benin 


\section{Introduction}

Dans le contexte actuel des changements globaux, les modifications spatio-temporelles de l'occupation des terres, et surtout celles qui concernent la végétation, sont devenues des indicateurs qui permettent d'évaluer la santé des écosystèmes [Arouna et al., 2016]. Ces modifications de la végétation sont dues aux phénomènes de déforestation et de dégradation des forêts, qui conduisent à un appauvrissement de la biodiversité [FAO, 2017 ; Ali, 2019 ; Ortega et al., 2019]. La « déforestation » est la conversion des forêts en d'autres unités d'occupation des terres, avec une diminution remarquable du taux de couverture de la canopée des arbres (Scouvart et Lambin, 2006) ; c'est le passage d'une classe «forêt » à une nouvelle classe «non-forêt ". "La notion de « dégradation des forêts " concerne l'ensemble des changements au sein de la forêt qui affectent négativement la structure ou la fonction du peuplement ou du site et qui, par conséquent, diminuent sa capacité à fournir des produits et/ou des services (FAO, 2017). Dans ce cas, le couvert forestier est toujours maintenu dans la classe "forêt ». L'exploitation abusive des habitats et des ressources biologiques, dans un contexte de faible application des textes réglementaires, constitue la cause principale de la dynamique spatiale des paysages naturels en Afrique de l'Ouest [Oloukoi et al., 2006 ; Hountondji, 2008].

Au Bénin, la dégradation des terres est un problème qui compromet aujourd'hui le développement et même la survie de la population. La dégradation du couvert végétal est de loin le phénomène le plus en vue. Elle est due à la culture itinérante sur brûlis, à la transhumance, à l'exploitation forestière (production de bois pour l'énergie domestique, les constructions et les meubles) et aux feux de végétation [Egah et al., 2014]. L'exploitation sans contrôle des écosystèmes forestiers à des fins agricoles et d'urbanisation, impriment une forte dynamique à l'occupation du sol et à l'utilisation des terres [Mama et al., 2020]. Selon Toko Imorou et al. [2019], les tendances évolutives des écosystèmes montrent une diminution de la superficie des formations denses, au profit de celles des savanes, des jachères et des espaces cultivés. Cette situation devient une préoccupation majeure, tant pour les institutions scientifiques que pour les gestionnaires des ressources naturelles au Bénin. Le Nord du Bénin en général et le département de l'Alibori en particulier, sont soumis, en plus des pratiques agricoles dégradantes, à une exploitation forestière intense et à des pratiques incontrôlées de feux de végétation. Ces pratiques dégradantes résultent des besoins socio-économiques d'une population à forte croissance et d'une urbanisation rapide [IFN, 2007; Mama et al., 2014]. Ces facteurs, associés aux pratiques de l'élevage extensif et transhumant de même qu'aux influences climatiques sahéliennes, entraînent une déforestation et une dégradation forestière galopantes dans cette région. Malheureusement, les aires protégées de cette région, qui devaient constituer une barrière verte à l'avancée du désert et protéger les bassins versants des rivières Mékrou, Alibori et Sota, sont de plus en plus empiétées par la production cotonnière et les activités pastorales [Mama et al., 2013]. Malgré les multiples recherches [Bogaert et al., 2011 ; Hountondji et al., 2013 ; Mama et al., 2013 ; Arouna et al., 2016 ; Kouta et al., 2018] réalisées dans cette zone pour alerter les décideurs et attirer l'attention des différents acteurs, rien ne semble freiner les dynamiques environnementales en cours. II est alors opportun de cartographier les changements spatiotemporels de l'occupation des terres dans ce milieu à partir de l'imagerie satellitaire.

L'imagerie satellitaire joue un rôle incontournable dans le processus de caractérisation et d'aménagement régional des écosystèmes (Maman et al., 2011). L'utilisation de la télédétection et des données géospatiales pour la cartographie de la végétation et de l'occupation des terres est une activité courante des institutions intéressées par la gestion des ressources végétales [Achard et al., 1996]. Cette approche cartographique a permis d'apprécier les changements de l'occupation des terres qui ont lieu dans le département de l'Alibori à travers les méthodes de détection des changements. La détection de changement est la mise en œuvre des techniques ayant pour but de repérer, de mettre en évidence, de quantifier afin de comprendre l'évolution temporelle ou le changement d'état d'un objet ou d'un phénomène à partir d'une série d'observations réalisées à différentes dates [Blanc, 1999 ; Fournier, 2008; Arouna, 2012]. II existe plusieurs méthodes de détection des changements de l'occupation des terres, à savoir la comparaison des signatures spectrales, la comparaison des indices de végétation et la comparaison des classifications, ou différence de classifications [Arouna, 2012]. Provencher-Nolet (2014) a fait un état de l'art détaillé des méthodes de détection des changements, en mettant en exergue les avantages et les inconvénients de chacune d'elles. Les méthodes de détection de changement répertoriées sont: l'analyse visuelle, la technique algébrique, la transformation à travers l'analyse en composantes principales, le modèle linéaire ou non linéaire de conversion des valeurs de réflectance, les techniques basées sur les systèmes d'information géographique (SIG) et la comparaison de classifications. Olaniyi et al. (2016) ont utilisé une méthode de détection multi-échelle basée sur les ondelettes avec des images radar. Afaq et Manocha (2021), ont également effectué un état de l'art des méthodes digitales de détection des changements. Ces derniers, ont conclu que la méthode d'analyse des vecteurs de changement offre une meilleure précision que l'approche de détection des changements basée sur l'algèbre. La comparaison des classifications permet de détecter les changements par le biais d'une table de contingence mettant en relation les couvertures de sol observées sur deux années différentes [Lu et al., 2004]. C'est pourquoi, cette dernière méthode a été préférée dans le cadre de la présente recherche. Ce choix est également justifié par le fait que toutes les images ne sont pas obtenues à partir des mêmes capteurs. Par ailleurs, cette méthode a l'avantage de diminuer l'impact des différences radiométriques entre les images, car ce sont les images déjà classifiées qui sont comparées [Coppin et al., 2004]. Cependant, la précision des résultats dépend de la qualité des interprétations, qui doit être

Revue Française de Photogrammétrie et Télédétection 
suffisamment élevée pour que les erreurs de détection ne soient pas propagées.

Afin d'obtenir des résultats de bonne qualité, la méthode d'interprétation numérique, complétée par une campagne de vérité-terrain et par une évaluation de la précision de la classification à travers la matrice de confusion, a été implémentée dans le cadre de cette recherche.

La présente recherche a pour objectif d'évaluer la déforestation et la dégradation des forêts à partir de l'imagerie satellitaire dans le département de l'Alibori au nord du Bénin, dans un contexte de changements environnementaux rapides observés de 2005 à 2015 . Cette recherche est fondée sur l'hypothèse qui stipule que le département de l'Alibori a enregistré un important phénomène de déforestation pendant cette période, aussi bien dans les terroirs villageois que les dans les aires protégées.

\section{Matériel et Méthodes}

\subsection{Cadre d'étude}

Le département de l'Alibori est localisé au nord-est du Bénin, entre $11^{\circ} 19^{\prime}$ et $12^{\circ} 24^{\prime}$ de latitude Nord, et entre $2^{\circ} 55^{\prime}$ et $3^{\circ} 50^{\prime}$ de longitude Est. II est limité au nord par la République du Niger, au nord-ouest par la République du Burkina Faso, à l'est par la République fédérale du Nigeria, à l'ouest par le département de l'Atacora et au sud par le département du Borgou. D'une superficie de $26242 \mathrm{~km}^{2}$ (23\% du territoire national), l'Alibori est subdivisé en six communes que sont Malanville, Karimama, Ségbana, Gogounou, Banikoara et Kandi, ce qui représente un total de 41 arrondissements, ainsi que de 229 villages et quartiers de villes (Figure 1). Quarante-deux pour cent de la superficie du département est occupée par des aires protégées, qui correspondent en l'occurrence aux zones cynégétiques de l'Atacora et de la Djona, aux forêts classées de l'Alibori Supérieur, aux Trois rivières, de la Sota, de Goungoun, de Kandi et de la Mékrou ainsi qu'au Parc national du W. La portion restante non occupée par les aires protégées est appelée terroirs villageois dans le cadre de cette recherche.

La population du département de l'Alibori est estimée à 867463 habitants en 2013 [INSAE, 2016] contre 521093 habitants en 2002 [INSAE, 2004]. Avec un taux d'accroissement de 4,61 entre 2002 et 2013 et un poids démographique national de $8,7 \%$, ce département affiche une densité de 33 habitants au $\mathrm{km}^{2}$. Dans l'Alibori, la population agricole représente $14 \%$ de la population agricole nationale, avec 74693 ménages agricoles sur 108 351 ménages. Ce département est le principal bassin cotonnier du Bénin. En plus du coton (Gossypium hirsutum), d'autres cultures vivrières comme le maïs (Zea mays), le sorgho (Sorghum bicolor) et le soja (Glycine max) sont cultivées et bénéficient des engrais utilisés pour la culture du coton.

Le climat du département de l'Alibori est de type soudanien au sud, mais évolue vers le type soudano-sahélien dans sa partie nord. II ne connaît qu'une seule saison des pluies, qui dure de cinq à six mois, avec une pluviosité oscillant entre $700 \mathrm{~mm}$ et $1200 \mathrm{~mm}$ [Vissin, 2007]. Cette pluviométrie donne naissance à plusieurs types de végétation : forêts galeries, forêts denses sèches, forêts claires, savanes boisées, arborées et arbustives. Les formations végétales naturelles dominantes sont: les savanes arbustives clairsemées, dominées par des épineux dont Acacia seyal et Acacia sieberiana au nord et les savanes arborées fortement dégradées au sud. On y trouve des arbres d'intérêts socioéconomiques, comme Parkia biglobosa (Néré), Vitellaria paradoxa (Karité) et Adansonia digitata (baobab), qui sont des essences protégées. En revanche, les galeries forestières sont éparses et longent les cours d'eau et les vallons. Ces forêts sont peuplées d'essences telles que Khaya senegalensis (Caïlcédrat), Khaya grandifolia (Faux acajou) et Afzelia africana (Lingué).

Le relief est dans son ensemble formé de plateaux qui peuvent être modelés dans une série sédimentaire du crétacé ou couronnés de buttes cuirassées qui descendent vers le fleuve Niger, ainsi que de collines de grès ferrugineux. On y rencontre trois types dominants de sols notamment les sols ferrugineux sur socle cristallin, les sols alluviaux très fertiles de la vallée du Niger et les sols argileux, limoneux noirs de bas-fonds, marécages et forêtgalerie très fertiles où se font la riziculture, le maraîchage et la culture de l'igname [Vissin, 2007].

\subsection{Données planimétriques}

Les données satellitaires utilisées sont les images SPOT de 2005 et pivots 2015 (2014, 2015, 2016). Ces différentes images fournies par le programme d'observation des forêts d'Afrique centrale et de l'Ouest (OSFACO) sont des données ortho-rectifiées en réflectance au sommet de l'atmosphère.

Au total, douze scènes d'image SPOT de 2005 et onze scènes d'images de 2015 ont été utilisées pour la cartographie de l'occupation des terres (tableau 1). 


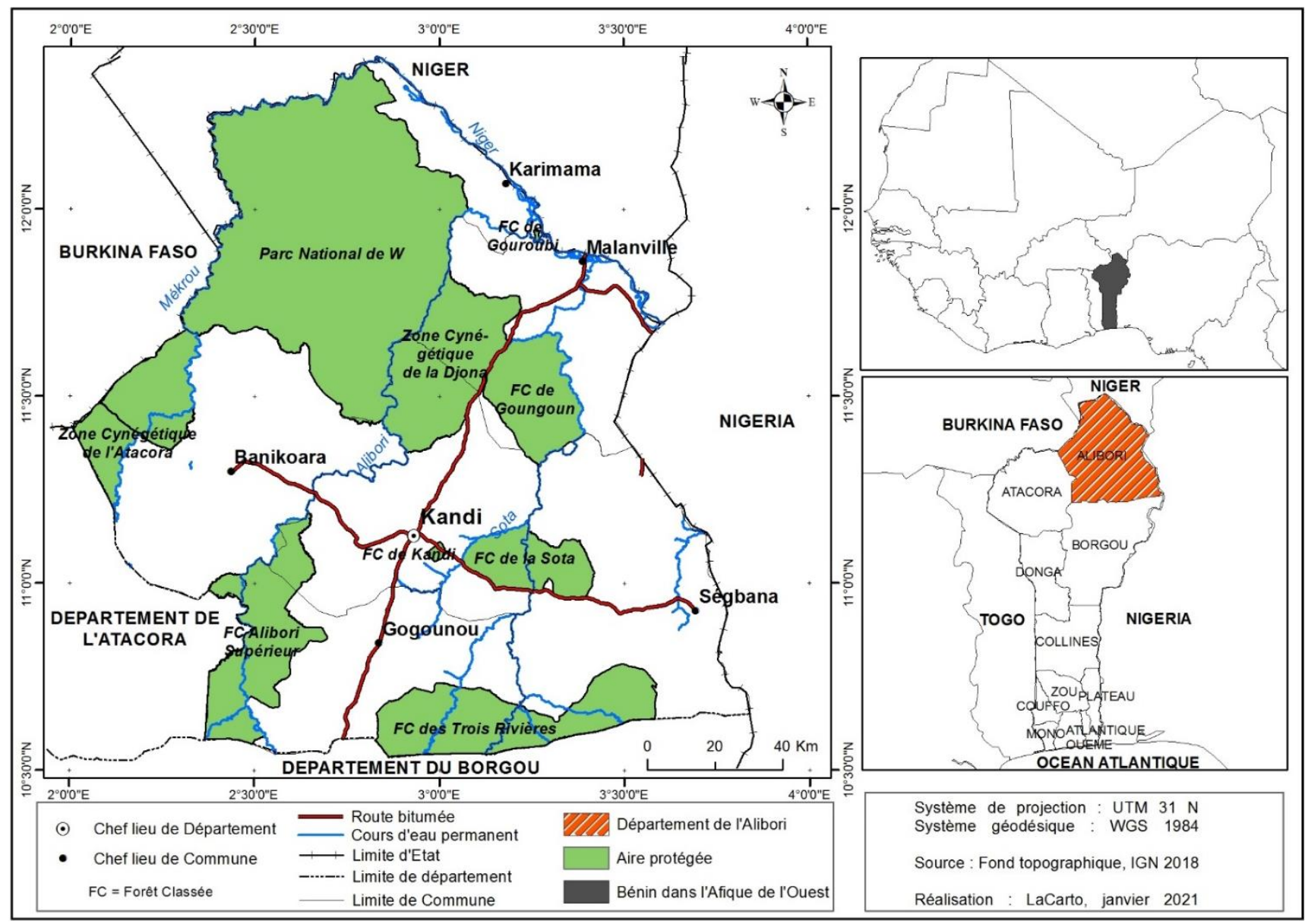

Figure 1 : Localisation du département de l'Alibori 


\begin{tabular}{|c|c|c|c|c|}
\hline $\mathrm{N}^{\circ}$ & Images & Date & Caractéristiques & Sources \\
\hline 1 & $\begin{array}{l}\text { SPOT5-HRG1- } \\
\text { XS en format Tiff }\end{array}$ & 09/10/2005 & $\begin{array}{l}\text { résolution spatiale de } \\
10 \mathrm{~m}(061-327)\end{array}$ & OSFACO \\
\hline 2 & $\begin{array}{l}\text { SPOT5-HRG1- } \\
\text { XS en format Tiff }\end{array}$ & $09 / 11 / 2005$ & $\begin{array}{l}\text { résolution spatiale de } \\
10 \mathrm{~m}(061-328)\end{array}$ & OSFACO \\
\hline 3 & $\begin{array}{l}\text { SPOT5-HRG1- } \\
\text { XS en format Tiff }\end{array}$ & $09 / 11 / 2005$ & $\begin{array}{c}\text { résolution spatiale de } \\
10 \mathrm{~m}(061-329)\end{array}$ & OSFACO \\
\hline 4 & $\begin{array}{l}\text { SPOT5-HRG1- } \\
\text { XS en format Tiff }\end{array}$ & $10 / 12 / 2005$ & $\begin{array}{c}\text { résolution spatiale de } \\
10 \mathrm{~m}(062-327)\end{array}$ & OSFACO \\
\hline 5 & $\begin{array}{l}\text { SPOT5-HRG1- } \\
\text { XS en format Tiff }\end{array}$ & $10 / 10 / 2005$ & $\begin{array}{c}\text { résolution spatiale de } \\
10 \mathrm{~m}(062-328)\end{array}$ & OSFACO \\
\hline 6 & $\begin{array}{l}\text { SPOT5-HRG1- } \\
\text { XS en format Tiff }\end{array}$ & $10 / 11 / 2005$ & $\begin{array}{c}\text { résolution spatiale de } \\
10 \mathrm{~m}(062-329)\end{array}$ & OSFACO \\
\hline 7 & $\begin{array}{l}\text { SPOT5-HRG1- } \\
\text { XS en format Tiff }\end{array}$ & 14/11/2005 & $\begin{array}{c}\text { résolution spatiale de } \\
10 \mathrm{~m}(064-327),\end{array}$ & OSFACO \\
\hline 8 & $\begin{array}{l}\text { SPOT } 5 \text { HRG en } \\
\text { format Tiff }\end{array}$ & $14 / 12 / 2005$ & $\begin{array}{l}\text { résolution spatiale de } \\
10 \mathrm{~m}(064-328),\end{array}$ & OSFACO \\
\hline 9 & $\begin{array}{l}\text { SPOT5-HRG1- } \\
\text { XS en format Tiff }\end{array}$ & $13 / 11 / 2005$ & $\begin{array}{c}\text { résolution spatiale de } \\
10 \mathrm{~m}(064-329),\end{array}$ & OSFACO \\
\hline 10 & $\begin{array}{l}\text { SPOT5-HRG1- } \\
\text { XS en format Tiff }\end{array}$ & 14/12/2005 & $\begin{array}{c}\text { résolution spatiale de } \\
10 \mathrm{~m}(065-327),\end{array}$ & OSFACO \\
\hline 11 & $\begin{array}{l}\text { SPOT5-HRG1- } \\
\text { XS en format Tiff }\end{array}$ & $14 / 12 / 2005$ & $\begin{array}{c}\text { résolution spatiale de } \\
10 \mathrm{~m}(065-328),\end{array}$ & OSFACO \\
\hline 12 & $\begin{array}{l}\text { SPOT5-HRG1- } \\
\text { XS en format Tiff }\end{array}$ & $14 / 12 / 2005$ & $\begin{array}{l}\text { résolution spatiale de } \\
10 \mathrm{~m}(065-329),\end{array}$ & OSFACO \\
\hline 13 & $\begin{array}{l}\text { SPOT } 7 \text { MS en } \\
\text { format JP2 }\end{array}$ & $07 / 11 / 2015$ & $\begin{array}{c}\text { résolution spatiale de } \\
6 \mathrm{~m}(0954-194),\end{array}$ & OSFACO \\
\hline 14 & $\begin{array}{l}\text { SPOT } 7 \text { MS en } \\
\text { format JP2 }\end{array}$ & $08 / 11 / 2015$ & $\begin{array}{l}\text { résolution spatiale de } \\
6 \mathrm{~m}(0946-225) .\end{array}$ & OSFACO \\
\hline 15 & $\begin{array}{l}\text { SPOT } 7 \text { MS en } \\
\text { format JP2 }\end{array}$ & $13 / 11 / 2015$ & $\begin{array}{c}\text { résolution spatiale de } \\
6 \mathrm{~m}(0957-191) .\end{array}$ & OSFACO \\
\hline 16 & $\begin{array}{l}\text { SPOT } 7 \text { MS en } \\
\text { format JP2 }\end{array}$ & 29/09/2014 & $\begin{array}{l}\text { résolution spatiale de } \\
6 \mathrm{~m}(0953-225) .\end{array}$ & OSFACO \\
\hline 17 & $\begin{array}{l}\text { SPOT } 7 \text { MS en } \\
\text { format JP2 }\end{array}$ & 29/09/2014 & $\begin{array}{l}\text { résolution spatiale de } \\
6 \mathrm{~m}(0953-490) .\end{array}$ & OSFACO \\
\hline 18 & $\begin{array}{l}\text { SPOT } 7 \text { MS en } \\
\text { format JP2 }\end{array}$ & $08 / 11 / 2014$ & $\begin{array}{l}\text { résolution spatiale de } \\
6 \mathrm{~m}(0946-225) .\end{array}$ & OSFACO \\
\hline 19 & $\begin{array}{l}\text { SPOT } 7 \text { MS en } \\
\text { format JP2 }\end{array}$ & $16 / 04 / 02016$ & $\begin{array}{l}\text { résolution spatiale de } \\
6 \mathrm{~m}(0958-240) .\end{array}$ & OSFACO \\
\hline 20 & $\begin{array}{l}\text { SPOT } 7 \text { MS en } \\
\text { format JP2 }\end{array}$ & 28/03/2016 & $\begin{array}{l}\text { résolution spatiale de } \\
6 \mathrm{~m}(0954-500) .\end{array}$ & OSFACO \\
\hline 21 & $\begin{array}{l}\text { SPOT } 7 \text { MS en } \\
\text { format JP2 }\end{array}$ & $28 / 02 / 2016$ & $\begin{array}{l}\text { résolution spatiale de } \\
6 \mathrm{~m}(0954-322) .\end{array}$ & OSFACO \\
\hline 22 & $\begin{array}{l}\text { SPOT } 7 \text { MS en } \\
\text { format JP2 }\end{array}$ & 29/12/2015 & $\begin{array}{c}\text { résolution spatiale de } \\
6 \mathrm{~m}(0946-325) .\end{array}$ & OSFACO \\
\hline 23 & $\begin{array}{l}\text { SPOT } 7 \text { MS en } \\
\text { format JP2 }\end{array}$ & $01 / 05 / 2015$ & $\begin{array}{c}\text { résolution spatiale de } \\
6 \mathrm{~m}(0957-107) .\end{array}$ & OSFACO \\
\hline
\end{tabular}

Sources : OSFACO, 2019

Tableau 1 : Caractéristiques des images couvrant le secteur d'étude

Les images SPOT-7 sont acquises à bord du satellite à 2 $\mathrm{m}$ de résolution (au nadir) pour le mode spectral panchromatique (noir et blanc) et à $8 \mathrm{~m}$ de résolution (au nadir) pour le mode multispectral (couleur) avec une plage dynamique de 12 bits par pixel. Elles sont ensuite rééchantillonnées au sol. La résolution des produits SPOT-
7 est de 1,5 m en mode panchromatique et de $6 \mathrm{~m}$ en mode multispectral. Les 4 bandes spectrales sont toujours acquises simultanément. II s'agit des bandes rouge (B1) : 0,625-0,695 $\mu \mathrm{m}$; vert (B2) : 0,530-0,590 $\mu \mathrm{m}$; bleu (B3) : $0,450-0,520 \mu \mathrm{m}$ : infrarouge proche (B4) : 0,760-0,890 $\mu \mathrm{m}$. 


\subsection{Outils de traitement}

Les outils suivants ont été utilisés pour le traitement des données.

- le logiciel QGIS Remote Sensing 2.18 muni d'Orfeo toolbox (OTB) pour le traitement des images satellites SPOT,

- le récepteur GPS pour le contrôle terrain,

- le plugin GEarthView for QGIS pour vérifier instantanément les classes douteuses sur la plateforme de Google Earth,

- le logiciel BaseCamp pour le déchargement des points GPS,

- le logiciel Google Earth Pro pour la vérification et le contrôle des unités à partir des images à très haute résolution.

Ces outils ont permis de réaliser les cartes d'occupation des terres du département de l'Alibori de 2005 et de 2015.

\subsection{Méthode de traitement des données}

La méthode adoptée est structurée en quatre étapes, à savoir : la composition colorée, le choix des aires d'entrainement, la classification supervisée et enfin la validation et la vectorisation de la classification.

\subsubsection{Composition colorée}

La composition colorée permet de produire des images en vraie couleur en tenant compte de la signature spectrale des objets. Elle sert avant tout à distinguer les différents objets présents dans les images et ainsi faciliter leur interprétation. La bande 4 (proche infrarouge), la bande 1 (rouge) et la bande 2 (vert) de SPOT 7 de 2015 ont été respectivement introduites dans les canaux du rouge, du vert et du bleu (RVB). Cette composition colorée, très efficace pour l'analyse de la végétation, présente l'avantage pour l'interprète d'avoir pratiquement les mêmes propriétés que les photographies infrarouges en couleur utilisées depuis longtemps par les photointerprètes [Kerle et Van Wyk De Vries, 2001]. Cette phase de composition colorée a été suivie du rehaussement du contraste de l'image (Figure 2).

II existe plusieurs techniques pour contraster une image. La méthode d'amélioration de contraste utilisée pour la présente recherche est l'égalisation d'histogramme. Avec cette méthode, les valeurs radiométriques d'image sont assignées aux grandes luminosités sur la base de leur fréquence d'occurrence, de sorte que les niveaux de luminosité les plus élevés soient assignés aux valeurs d'image qui se produisent fréquemment [Toko Mouhamadou, 2014]. Cette technique a permis de rendre l'image plus lisible et facilement interprétable.

\subsubsection{Choix des aires d'entraînement}

Les aires d'entraînement sont des sites représentatifs des caractéristiques numériques des classes qui permettent de définir les signatures spectrales de chaque unité paysagère. La taille de l'aire d'entraînement est supérieure à l'erreur de localisation et inférieure à l'objet à détecter [Kioko et Okello, 2010].

Elle est estimée de la façon suivante : $A=P(1+2 L)(1)$ [Arouna, 2012], avec $A=$ la superficie de l'aire d'entraînement, $P=$ dimension du pixel en mètre, $L=$ précision de la localisation en mètre.

Sur les images, les aires d'entraînement ont été tracées au pixel près. Ces aires ont été bien dispersées sur l'ensemble du département et sont représentatives de la diversité de chaque classe de végétation ou autre unité d'occupation des terres. En effet, quinze aires d'entraînement ont été choisies au niveau de chaque classe de végétation naturelle (forêts, savanes, etc.), contre dix pour les cultures et jachères et cinq pour chacune des autres classes d'occupation des terres. Au total, 100 aires d'entraînement ont été échantillonnées à l'échelle du secteur d'étude pour les douze classes définies. Les aires d'entraînement ont été choisies de manière indépendante pour chaque date, car les images SPOT 5 de 2005 sont obtenues en fausse couleur avec trois bandes (RVB). Ces aires d'entraînement ont été validées sur la base des images Google Earth.

\subsubsection{Classification supervisée}

La classification supervisée est une classification pixel par pixel qui repose sur le postulat que la signature spectrale de chacun des pixels est représentative de la classe de végétation dans laquelle il se trouve. Elle a consisté à attribuer à chaque groupe de pixels la classe la plus plausible en fonction de la ressemblance spectrale entre les pixels et la signature des classes. L'ensemble des pixels de chaque image satellite a été classé suivant l'algorithme du maximum de vraisemblance qui extrapole les caractéristiques spectrales des aires d'entraînement au reste de l'image. Les pixels qui n'ont pas pu être affectés à une classe de végétation ont été classés en rejet, puis identifiés au cours du contrôle-terrain. Celui-ci a consisté à vérifier les classes de pixels issues de la classification, et à valider l'interprétation de l'image. 


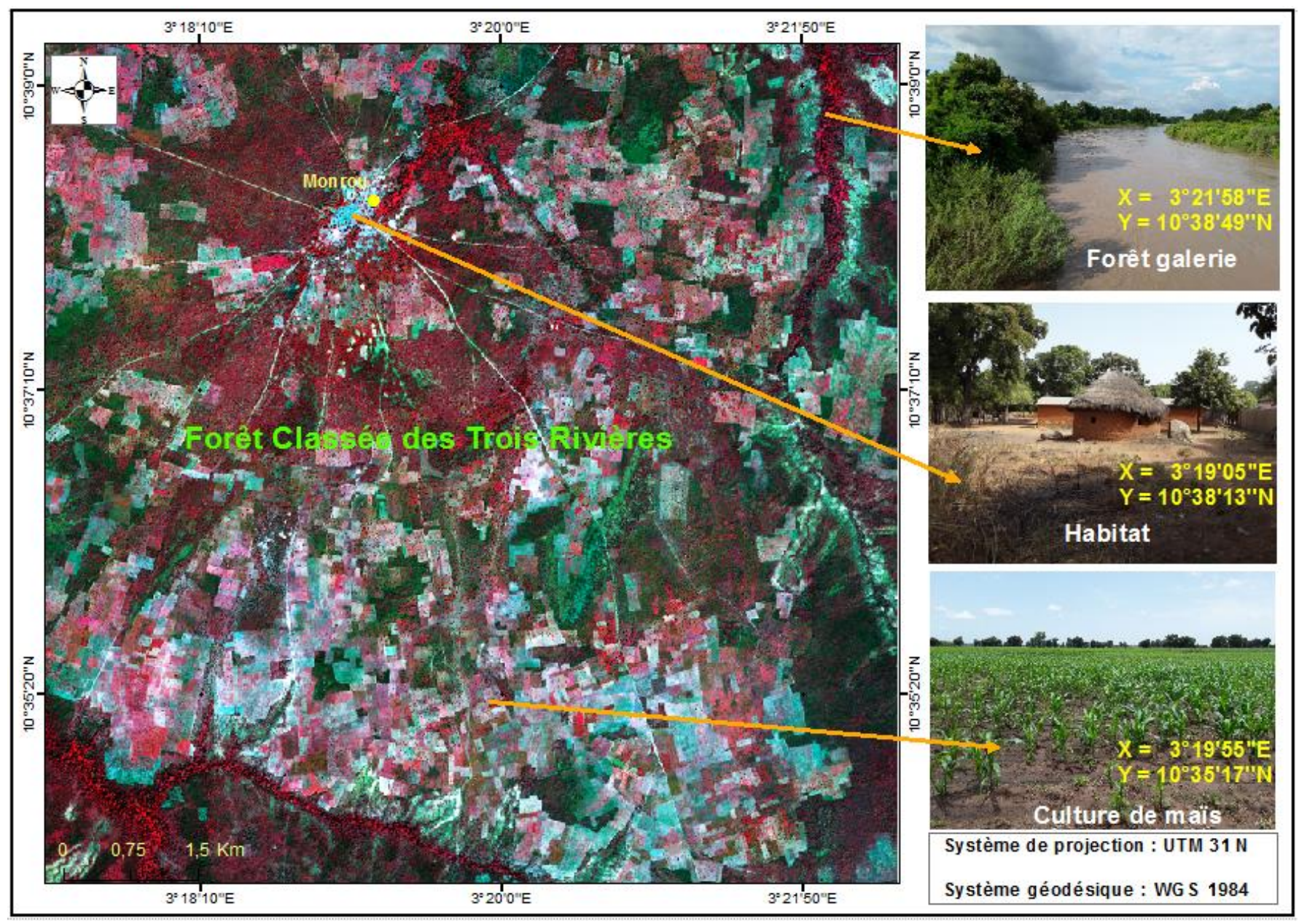

Figure 2 : Composition colorée 412 de l'image SPOT 7 de 2015 et quelques points de contrôle de terrain 


\subsubsection{Validation et vectorisation de la classification}

L'évaluation de la qualité de la classification a été suivie du contrôle-terrain qui a consisté, d'une part en la vérification sur le terrain des interprétations effectuées, et d'autre part à collecter des informations sur les formations végétales, la toponymie ainsi que d'autres informations socioéconomiques.

La démarche utilisée pour le contrôle et la vérité terrain est basée sur le ralliement systématique de 250 points stations à partir de leurs coordonnées géographiques à l'aide d'un GPS «Garmin Etrex 10 » dont est utilisée la fonction "Go to ». Ces points stations sont localisés sur les principaux axes routiers du secteur d'étude et sont placés à une distance de $5 \mathrm{~km}$ les uns des autres. Un point de contrôle terrain est marqué de part et d'autre, à environ $200 \mathrm{~m}$ de chaque point station (soit 500 points de contrôle pour 250 points stations) de façon aléatoire. De plus, les coordonnées de 52 points ont été relevées et vérifiées sur le terrain, au niveau des unités qui ont prêté à confusion lors de l'interprétation. Par ailleurs, 300 autres points complémentaires sont enregistrés aléatoirement au cours du trajet pour des classes spécifiques, afin d'inclure certains détails essentiels, tels que les couloirs de passage, les campements peulh, les équipements et infrastructures communautaires (barrage, surcreusement, etc.) ou encore les bosquets. Les points de contrôle terrain sont ensuite exportés du GPS vers un SIG (QGIS) en format shapefile (SHP) à l'aide du logiciel BaseCamp.

Au total, 852 points de contrôle ont été enregistrés (tableau 2). Les images Google Earth ont aussi été utilisées pour confirmer ou infirmer certaines classes d'occupation des terres de façon interactive à partir de QGIS, en utilisant le plugin GEarthView for QGIS.

Après l'intégration des observations de terrain et de la correction de la classification, chaque image interprétée a été vectorisée. Cela a été fait à l'aide de l'extension " conversion raster to vector » du logiciel QGIS Remote Sensing 2.18.

En effet, la matrice de confusion a permis d'évaluer la qualité de l'interprétation. Elle compare les données interprétées (colonne) avec les données de vérité terrain (ligne). La diagonale représente la concordance entre l'interprétation et les données de référence terrain. Cette matrice de confusion a permis de calculer les erreurs d'omission (EO), les erreurs de commission (EC), les indices de pureté des classes (IPC) et la précision globale de l'interprétation. La précision globale est l'ensemble de toutes les classes d'occupation des terres interprétées qui sont conformes à la réalité de terrain. Elle est calculée à l'aide de la formule suivante :

$a=\frac{1}{N} \sum_{i=1}^{N c} x_{i i}$

Dans cette formule, a est la précision globale, Nc le nombre total de classe, $x_{i i}$ nombre de points d'observation dans la colonne $i$ et la ligne $i$

$N$ est le nombre total des observations pour l'ensemble des classes.

\subsection{Analyse quantitative de la déforestation}

La quantification de la déforestation a été faite à partir du calcul du taux global de changement, du taux de déforestation, du taux de dégradation, du taux d'amélioration des formations végétales naturelles et du taux de reconstitution naturelle. Ces calculs sont essentiellement basés sur la matrice de conversion des unités entre 2005 et 2015, qui a été générée.

Pour faciliter les analyses, les différentes unités d'occupation des terres ont été regroupées en trois catégories que sont les terres forestières (TF), les terres cultivées (TC) et les autres terres (AT).

La nomenclature à douze postes retenue a connu un réaménagement. Les douze classes ont été agrégées en trois grandes catégories d'affectation des terres, à savoir : les terres forestières (FGFR, FD, FCSB, SASa, FSM), les terres cultivées (PTFR, PTFT, CJ) et les autres terres (PE, HA, SR, SED).

La déforestation est donc le passage de «terres forestières » aux « terres non forestières » que sont les terres cultivées et les autres terres (PTFR, PTFT, CJ, PE, HA, SR, SED), selon le guide des bonnes pratiques du GIEC (2003) ainsi que d'après les lignes directrices sur le secteur de l'agriculture, de l'utilisation des terres et de la forêt (GIEC, 2006)

Le tableau 2 montre la matrice d'analyse de la déforestation et de la dégradation.

La déforestation et la dégradation ont été analysées à travers le calcul des taux globaux et annuels de déforestation brute et nette, de dégradation brute et nette, et de reconstitution naturelle et d'amélioration.

Le taux global de déforestation Tg (Def) est calculé par la formule de De Wasseige et al. (2008) utilisée par Tchatchou et al. (2015) et Issifou Moumouni (2020). 


\begin{tabular}{|c|c|c|c|c|c|c|c|c|c|c|c|c|}
\hline \multirow{2}{*}{ Unités } & \multicolumn{5}{|c|}{ Terres Forestières (TF) } & \multicolumn{3}{|c|}{ Terres cultivées (TC) } & \multicolumn{4}{|c|}{ Autres terres (AT) } \\
\hline & FD & FGFR & FCSB & FSM & SASa & PTFR & PTFT & CJ & $\mathrm{PE}$ & HA & SR & SED \\
\hline FD & S & $\mathrm{Dg}$ & $\mathrm{Dg}$ & $\mathrm{Dg}$ & $\mathrm{Dg}$ & $\operatorname{Re}$ & $\operatorname{Re}$ & Def & Def & Def & Def & Def \\
\hline FGFR & $\mathrm{Am}$ & S & $\mathrm{Dg}$ & $\mathrm{Dg}$ & $\mathrm{Dg}$ & $\mathrm{Re}$ & $\mathrm{Re}$ & Def & Def & Def & Def & Def \\
\hline FCSB & $\mathrm{Am}$ & $\mathrm{Am}$ & $\mathrm{S}$ & $\mathrm{Dg}$ & $\mathrm{Dg}$ & $\mathrm{Re}$ & $\mathrm{Re}$ & Def & Def & Def & Def & Def \\
\hline FSM & $\mathrm{Am}$ & $\mathrm{Am}$ & $A m$ & $\mathrm{~S}$ & $\mathrm{Dg}$ & $\mathrm{Re}$ & $\mathrm{Re}$ & Def & Def & Def & Def & Def \\
\hline SASa & $\mathrm{Am}$ & $A m$ & $\mathrm{Am}$ & $\mathrm{Am}$ & S & $\mathrm{Re}$ & $\mathrm{Re}$ & Def & Def & Def & Def & Def \\
\hline $\begin{array}{l}\text { PTFR } \\
\end{array}$ & $\mathrm{Rn}$ & $\mathrm{Rn}$ & $\mathrm{Rn}$ & $\mathrm{Rn}$ & $\mathrm{Rn}$ & $\mathrm{S}$ & $\mathrm{bBa}$ & $\mathrm{bBa}$ & $\mathrm{bBa}$ & $\mathrm{bBa}$ & $\mathrm{bBa}$ & $\mathrm{bBa}$ \\
\hline PTFT & $\mathrm{Rn}$ & $\mathrm{Rn}$ & $\mathrm{Rn}$ & $\mathrm{Rn}$ & $\mathrm{Rn}$ & $\mathrm{Re}$ & $\mathrm{S}$ & $\mathrm{bBa}$ & $\mathrm{bBa}$ & $\mathrm{bBa}$ & $\mathrm{bBa}$ & $\mathrm{bBa}$ \\
\hline CJ & $\mathrm{Rn}$ & $\mathrm{Rn}$ & $\mathrm{Rn}$ & $\mathrm{Rn}$ & $\mathrm{Rn}$ & $\operatorname{Re}$ & $\operatorname{Re}$ & S & $\mathrm{bBa}$ & $\mathrm{bBa}$ & $\mathrm{bBa}$ & $\mathrm{bBa}$ \\
\hline $\mathrm{PE}$ & $\mathrm{Rn}$ & $\mathrm{Rn}$ & $\mathrm{Rn}$ & $\mathrm{Rn}$ & $\mathrm{Rn}$ & $\mathrm{Re}$ & $\mathrm{Re}$ & $\mathrm{aBa}$ & $\mathrm{S}$ & $\mathrm{bBa}$ & $\mathrm{bBa}$ & $\mathrm{bBa}$ \\
\hline HA & $\mathrm{Rn}$ & $\mathrm{Rn}$ & $\mathrm{Rn}$ & $\mathrm{Rn}$ & $\mathrm{Rn}$ & $\operatorname{Re}$ & $\operatorname{Re}$ & $\mathrm{aBa}$ & $\mathrm{aBa}$ & $\mathrm{s}$ & $\mathrm{bBa}$ & $\mathrm{bBa}$ \\
\hline SR & $\mathrm{Rn}$ & $\mathrm{Rn}$ & $\mathrm{Rn}$ & $\mathrm{Rn}$ & $\mathrm{Rn}$ & $\operatorname{Re}$ & $\operatorname{Re}$ & $\mathrm{aBa}$ & $\mathrm{aBa}$ & $\mathrm{aBa}$ & $\mathrm{s}$ & $\mathrm{bBa}$ \\
\hline SED & $\mathrm{Rn}$ & $\mathrm{Rn}$ & $\mathrm{Rn}$ & $\mathrm{Rn}$ & $\mathrm{Rn}$ & $\mathrm{Re}$ & $\mathrm{Re}$ & $\mathrm{aBa}$ & $\mathrm{aBa}$ & $\mathrm{aBa}$ & $\mathrm{aBa}$ & S \\
\hline \multicolumn{13}{|c|}{$\begin{array}{l}\text { FD : Forêt dense, FGFR: Forêt galerie et formation ripicole, FCSB : Forêt claire et savane boisée, FSM : Forêt et savane } \\
\text { marécageuse, SASa: Savanes arborée et arbustive, PTFR: Plantation forestière, PTFT: Plantation fruitière, CJ : Culture e } \\
\text { jachère, PE: Plan d'eau, HA : Habitat, SR : Surface rocheuse, SED : Sol érodé et dénudé. }\end{array}$} \\
\hline Def_brute & \multicolumn{12}{|c|}{ Déforestation brute : conversion des terres forestières en terre cultivée et autres terres } \\
\hline Dg_brute & \multicolumn{12}{|c|}{$\begin{array}{l}\text { Dégradation brute : conversion d'une terre forestière dense ou fermée en une terre forestière moins dense ou } \\
\text { ouverte }\end{array}$} \\
\hline Am & \multicolumn{12}{|c|}{ Amélioration : conversion d'une terre forestière ouverte en une terre forestière plus fermée ou dense } \\
\hline $\mathrm{Rn}$ & \multicolumn{12}{|c|}{ Reconstitution naturelle : conversion des terres cultivées et autres terres en terres forestières } \\
\hline $\operatorname{Re}$ & \multicolumn{12}{|c|}{ Reboisement : conversion des terres forestières, terres cultivées (CJ) et autres terres en plantations } \\
\hline $\mathrm{bBa}$ & \multicolumn{12}{|c|}{ Baisse de la biomasse des autres terres } \\
\hline $\mathrm{aBa}$ & \multicolumn{12}{|c|}{ Augmentation de la biomasse des autres terres } \\
\hline S & \multicolumn{12}{|l|}{ Stabilité } \\
\hline Def_nette & \multicolumn{12}{|c|}{ Déforestation nette $=$ déforestation brute - reconstitution naturelle } \\
\hline Dg_nette & \multicolumn{12}{|c|}{ Dégradation nette $=$ dégradation - amélioration } \\
\hline
\end{tabular}

Tableau 2 : Matrice d'analyse de la déforestation et de la dégradation

Source : FAO, 2016

Les différentes valeurs brutes de la déforestation, de la dégradation, de la reconstitution naturelle et des améliorations sont déduites de la matrice d'analyse du tableau 2 en s'inspirant de De Wasseige et al. (2008)

\subsubsection{Taux global de changement}

Soit $\mathrm{S} 1$, la superficie d'une formation végétale à la date t1 (2005) ; soit S2, la superficie de la même formation à la date t2 (2015). Le taux de changement global (Tg) utilisé par Arouna (2012), est obtenu par la formule :

$\mathrm{Tg}=\frac{\mathrm{S} 2-\mathrm{S} 1}{\mathrm{~S} 1} \times 100$

\subsubsection{Taux de déforestation}

La déforestation est le passage de " terre forestière " à " terre non forestière ". La déforestation brute est obtenue par la somme des conversions de la catégorie «terre forestière » en catégories "terres cultivées, autres terres ». Une classe de reconstitution naturelle a été distinguée, ce qui a permis de calculer un taux de déforestation net en soustrayant la reconstitution naturelle de la déforestation brute.

Le taux annuel de déforestation a été obtenu en divisant le taux de déforestation par le nombre d'années.

$\operatorname{Tg}($ Def $)=\frac{\text { Def } \times 100}{S 1}$

$\operatorname{Def}(b ; n)$ est la déforestation brute (b) ou nette (n) et S1 représente la superficie des terres forestières naturelles de l'année $\mathrm{t} 1$.

\subsubsection{Taux de dégradation}

La dégradation brute est obtenue par la somme des transitions de la catégorie " terre forestière » en « terre forestière clairsemée » (plus ouverte), qui diminue sa capacité de production de biens et services et surtout de séquestration du carbone [Issifou Moumouni, 2020]. Le passage de «forêt dense » à « forêt clairsemée » peut parfois concerner des espèces décidues qui perdent en densité foliaire en saison sèche. Ce type de dégradation naturelle n'est pas concerné par la présente recherche, car toutes les images satellites utilisées ont été prises en saison sèche.

Le taux global de dégradation $\mathrm{Tg}(\mathrm{Dg})$ est calculé par la formule suivante :

$\operatorname{Tg}(\mathrm{Dg})=\frac{\mathrm{Dg} \times 100}{\mathrm{~S} 1}$

$\mathrm{Dg}(\mathrm{b} ; \mathrm{n})$ est la dégradation brute (b) ou nette $(\mathrm{n})$ et $\mathrm{S} 1$ représente la superficie des terres forestières naturelles de l'année t1.

\subsubsection{Taux de reconstitution naturelle}

$\mathrm{La}$ «reconstitution» est la transition d'une «terre cultivée » ou « autres terres » en « terres forestières ». Le taux global de reconstitution naturelle $\mathrm{Tg}(\mathrm{Rn})$ est obtenu par la formule suivante [Issifou Moumouni, 2020]

$$
\operatorname{Tg}(\mathrm{Rn})=\frac{\operatorname{Rn} \times 100}{\mathrm{~S} 1}
$$


Rn est la reconstitution naturelle totale enregistrée dans la période et $\mathrm{S} 1$ représente la superficie des terres forestières naturelles de l'année $t 1$.

\subsubsection{Taux d'amélioration}

L'amélioration est la conversion d'une terre forestière ouverte en une terre forestière plus fermée ou dense [Issifou Moumouni, 2020]. Son taux global $\mathrm{Tg}(\mathrm{Am})$ est : $\mathrm{Tg}(\mathrm{Am})=\frac{\mathrm{Am} \times 100}{\mathrm{~S} 1}$

Am est l'amélioration totale enregistrée dans la période et $S 1$ représente la superficie des terres forestières naturelles de l'année t1. Le taux annuel a été obtenu en divisant le taux global par le nombre d'années d'étude.

\section{Résultats}

\section{1 État de l'occupation des terres dans le} département de l'Alibori entre 2005 et 2015

La matrice de confusion issue du traitement des données de vérité terrain indique une précision globale de la carte de $96 \%$ (Tableau 3).

La figure 3 présente les états de l'occupation des terres entre 2005 et 2015.

L'analyse de l'occupation des terres entre 2005 et 2015 révèle la diminution des formations végétales naturelles qui dominaient la physionomie du paysage en 2005.

\subsection{Changement d'état des unités d'occupation des terres}

Le tableau 4 présente la matrice de transition des unités d'occupation des terres de 2005 à 2015.

L'examen de la matrice de transition (Tableau 4) révèle que toutes les unités d'occupation des terres observées en 2005 sont également présentes en 2015. De façon générale, on remarque que les formations naturelles ont régressé en subissant deux modes de conversion : la savanisation et l'anthropisation.

La matrice de transition établie pour la période 2005 à 2015 permet de mieux appréhender les différentes conversions qui ont lieu au sein de chaque unité d'occupation des terres.

Elles ont laissé place aux champs et jachères aussi bien dans les terroirs villageois que dans les aires protégées qui constituent la principale unité paysagère du milieu en 2015. Les formations forestières naturelles résiduelles s'observent seulement dans les aires protégées.

En effet, les forêts denses, les forêts claires et savanes boisées qui occupaient respectivement $0,12 \%$ et 8,06\% en 2005 sont passés à $0,07 \%$ et $4,27 \%$ en 2015 . Une partie de ces unités s'est convertie en savanes arborée et arbustive, soit $16,67 \%$ et $31,44 \%$ respectivement.

Pour ce qui concerne le second mode qu'est l'anthropisation, ce sont les forêts denses $(20,83 \%)$, les forêts galeries $(11,22 \%)$, les forêts claires et savanes boisées $(19,22 \%)$, les forêts et savanes marécageuses $(31,50 \%)$ ainsi que les savanes arborée et arbustive $(20,62 \%)$ qui ont été converties respectivement en cultures et jachères. Ces dernières ont aussi cédé une partie de leur superficie aux habitats humains $(0,05 \%)$.

\section{3 Évolution temporelle des catégories d'occupation des terres}

Pour faciliter les analyses de la dynamique de l'occupation des terres, les différentes unités de l'occupation des terres ont été regroupées en trois catégories (Figure 4).

\begin{tabular}{|c|c|c|c|c|c|c|c|c|c|c|c|c|c|c|c|}
\hline \multirow{2}{*}{$\begin{array}{l}\text { Unités observées } \\
\text { sur le terrain }\end{array}$} & \multicolumn{15}{|c|}{ Classification } \\
\hline & FD & FGFR & FCSB & FSM & SASa & PTFR & PTFT & CJ & PE & HA & SR & SED & TOTAL & $\begin{array}{l}\text { EC } \\
\text { (\%) }\end{array}$ & $\begin{array}{l}\text { IPC } \\
\text { (\%) }\end{array}$ \\
\hline FD & 37 & 1 & 0 & 0 & 0 & 0 & 0 & 0 & 0 & 0 & 0 & 0 & 38 & $97 \%$ & $3 \%$ \\
\hline FGFR & 0 & 68 & 0 & 0 & 0 & 1 & 1 & 0 & 0 & 0 & 0 & 0 & 70 & $97 \%$ & $3 \%$ \\
\hline FCSB & 0 & 1 & 75 & 1 & 0 & 0 & 0 & 0 & 0 & 0 & 0 & 0 & 77 & $97 \%$ & $3 \%$ \\
\hline FSM & 0 & 0 & 1 & 164 & 3 & 0 & 0 & 1 & 0 & 0 & 0 & 0 & 169 & $97 \%$ & $3 \%$ \\
\hline SASa & 0 & 0 & 0 & 1 & 25 & 1 & 1 & 0 & 0 & 0 & 0 & 0 & 28 & $89 \%$ & $11 \%$ \\
\hline PTFR & 0 & 0 & 0 & 0 & 0 & 64 & 7 & 1 & 0 & 0 & 0 & 0 & 72 & $89 \%$ & $11 \%$ \\
\hline PTFT & 0 & 0 & 0 & 0 & 1 & 5 & 59 & 1 & 0 & 0 & 0 & 0 & 66 & $8 \%$ & $92 \%$ \\
\hline CJ & 0 & 1 & 0 & 1 & 1 & 0 & 0 & 145 & 0 & 0 & 0 & 0 & 148 & $98 \%$ & $2 \%$ \\
\hline PE & 0 & 0 & 0 & 0 & 0 & 0 & 0 & 0 & 27 & 0 & 0 & 0 & 27 & $100 \%$ & $0 \%$ \\
\hline HA & 0 & 0 & 0 & 0 & 0 & 0 & 0 & 0 & 0 & 51 & 0 & 1 & 52 & $98 \%$ & $2 \%$ \\
\hline SR & 0 & 0 & 0 & 0 & 0 & 0 & 0 & 0 & 0 & 0 & 32 & 0 & 32 & $100 \%$ & $0 \%$ \\
\hline SED & 0 & 0 & 0 & 0 & 0 & 0 & 0 & 0 & 0 & 0 & 1 & 72 & 73 & $99 \%$ & $1 \%$ \\
\hline TOTAL & 37 & 71 & 76 & 167 & 30 & 71 & 68 & 148 & 27 & 51 & 33 & 73 & 852 & & \\
\hline $\begin{array}{l}\text { Précision } \\
\text { producteur }\end{array}$ & $100 \%$ & $96 \%$ & $99 \%$ & $98 \%$ & $83 \%$ & $7 \%$ & $87 \%$ & $98 \%$ & $100 \%$ & $100 \%$ & $97 \%$ & $99 \%$ & & & \\
\hline Omission (\%) & $0 \%$ & $4 \%$ & $1 \%$ & $2 \%$ & $17 \%$ & $93 \%$ & & $2 \%$ & $0 \%$ & $0 \%$ & $3 \%$ & $1 \%$ & & & \\
\hline Précision globale : & & & & & & & & & & & & & $96 \%$ & & \\
\hline
\end{tabular}

FD : Forêt dense, FGFR : Forêt galerie et formation ripicole, FCSB : Forêt claire et savane boisée, SASa : Savanes arborée et arbustive, FSM : Forêt et savane marécageuse, PTFR : Plantation forestière, PTFT : fruitière, CJ : Culture et jachère, CJP: Culture et jachère sous palmier, PE: Plan d'eau, HA: Habitat, SR: Surface rocheuse, SED: sol érodé et dénudé, SS : Surface sableuse ; IPC : Indice de Pureté des Classes, EC : Erreur de commission

Sources : Données de terrain, 2015, Interprétation SPOT7

Tableau 3 : Matrice de confusion 


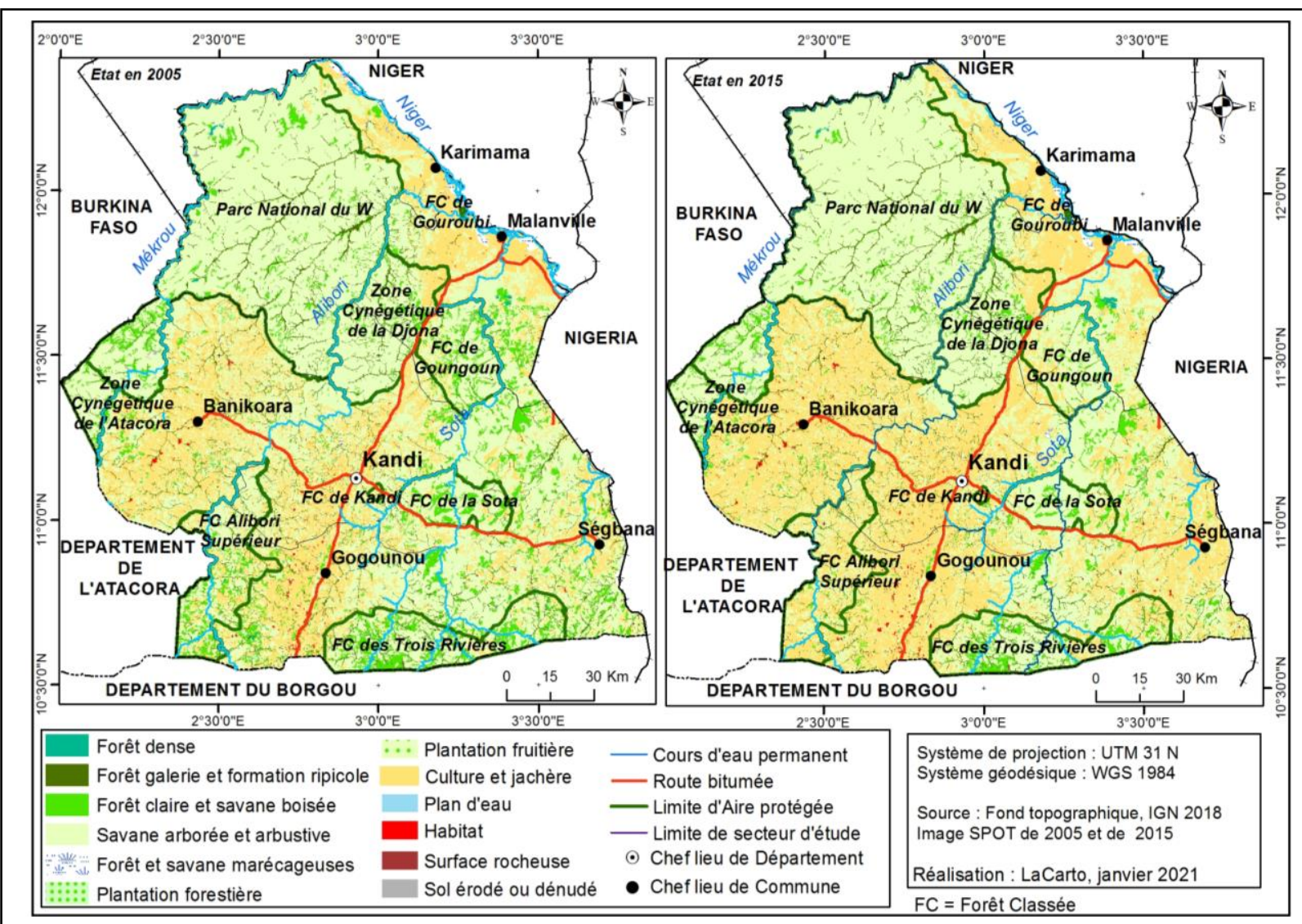

Figure 3 : État de l'occupation des terres dans le département de l'Alibori en 2005 et 2015

La figure 4 présente l'évolution temporelle des unités d'occupation des terres aussi bien dans les aires protégées que dans les terroirs villageois (ensemble des terres hors des aires protégées).

II ressort de l'analyse de la figure 4 que, les formations forestières naturelles ont perdu en moyenne $1,83 \%$, soit environ 35354 ha / an dans tout le département de l'Alibori. Ce taux est plus élevé dans les terroirs villageois (3,10\%, soit, 28377 ha / an) et plus ou moins faible dans les aires protégées $(0,70 \%$ soit, 7079 ha / an).

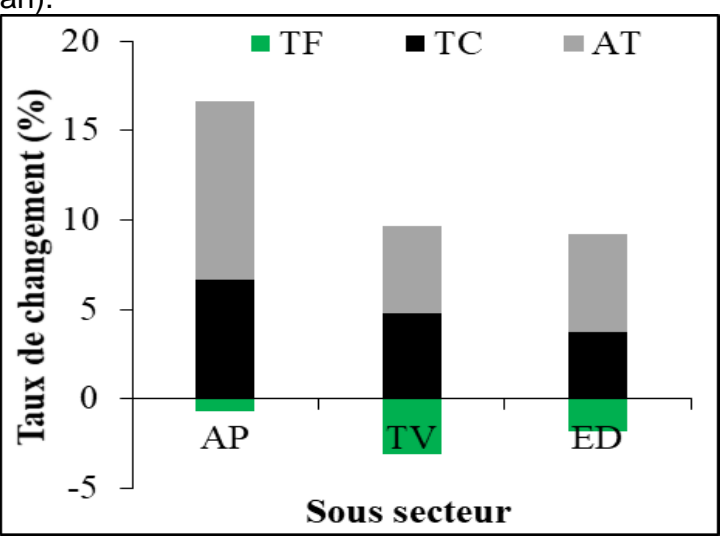

Légende : $E D$ : ensemble du département; $T V$ : terroirs villageois ; $A P$ : aire protégée, $T F$ : terres forestières, $T C$ : terres cultivées, $A T$ : autres terres

Figure 4 : Taux global de changement des formations forestières naturelles, des plantations et autres formations non forestières entre 2005 et 2015

On note par ailleurs une progression des plantations et des autres formations non forestières avec un fort taux dans les aires protégées ( $10 \%$ soit, 7060 ha / an).
Le fort taux observé au niveau des autres formations non forestières s'explique par l'extension des zones de culture, et cela particulièrement dans les forêts classées de Goungoun, de la Sota et de l'Alibori supérieur.

\subsection{Analyse des changements d'occupation des terres entre 2005 et 2015}

La figure 5 présente les pertes et les gains observés entre les différentes catégories d'occupation des terres et à l'intérieur de chacune d'elles dans le département entre 2005 et 2015.

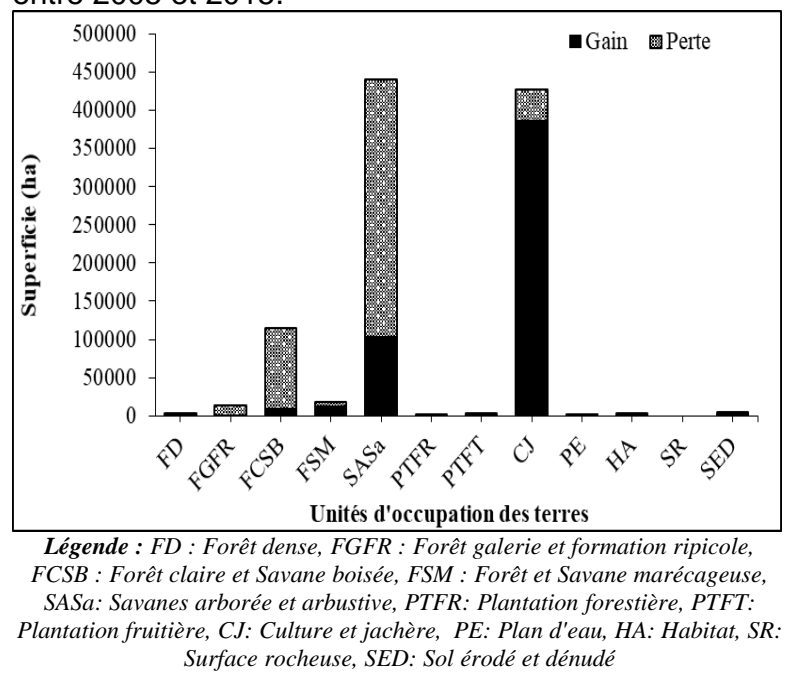

Figure 5 : Gains et pertes de superficie d'occupation des terres de 2005 à 2015 


\begin{tabular}{|c|c|c|c|c|c|c|c|c|c|c|c|c|c|}
\hline \multirow{2}{*}{ UOT 2005} & \multicolumn{12}{|c|}{ UOT 2015} & \multirow{2}{*}{ Total 2005} \\
\hline & FD & \begin{tabular}{|l|} 
FGFR \\
\end{tabular} & FCSB & FSM & SASa & \begin{tabular}{|l|l|} 
PTFR \\
\end{tabular} & PTFT & CJ & PE & HA & SR & SED & \\
\hline FD & 1221 & 0 & 707 & 0 & 514 & 0 & 0 & 643 & 0 & 0 & 0 & 0 & 3085 \\
\hline FGFR & 0 & 106108 & 0 & 129 & 0 & 0 & 64 & 13432 & 0 & 0 & 0 & 0 & 119733 \\
\hline FCSB & \begin{tabular}{|l|}
643 \\
\end{tabular} & 0 & 102059 & 0 & 65490 & 0 & 0 & 40040 & 0 & 0 & 0 & 64 & 208296 \\
\hline FSM & 0 & 0 & 0 & 11890 & 0 & 0 & 0 & 5527 & 129 & 0 & 0 & 0 & 17545 \\
\hline SASa & 0 & 0 & 7584 & 0 & 1247591 & 64 & 257 & 326937 & 0 & 900 & 0 & 2314 & 1585646 \\
\hline \begin{tabular}{|l|} 
PTFR \\
\end{tabular} & 0 & 0 & 0 & 0 & 0 & 450 & 0 & 0 & 0 & 0 & 0 & 0 & 450 \\
\hline PTFT & 0 & 0 & 0 & 0 & 0 & 0 & 4306 & 64 & 0 & 0 & 0 & 0 & 4370 \\
\hline CJ & 0 & 0 & 0 & 257 & 36248 & 0 & \begin{tabular}{|l|}
1478 \\
\end{tabular} & 584270 & 0 & 2699 & 0 & 129 & 625081 \\
\hline PE & 0 & 0 & 0 & 0 & 0 & 0 & 0 & 64 & 5334 & 0 & 0 & 0 & 5399 \\
\hline $\mathrm{HA}$ & 0 & 0 & 0 & 0 & 0 & 0 & 0 & 0 & 0 & 10155 & 0 & 0 & 10155 \\
\hline SR & 0 & 0 & 0 & 0 & 0 & 0 & 0 & 0 & 0 & 0 & 386 & 0 & 386 \\
\hline SED & 0 & 0 & 0 & 0 & 321 & 0 & 0 & 64 & 0 & 0 & 0 & 4884 & 5270 \\
\hline \begin{tabular}{|l|} 
Total 2015 \\
\end{tabular} & 1864 & 106108 & 110350 & 12275 & 1350164 & 514 & 6106 & 971041 & 5463 & 13754 & 386 & 7391 & 2585416 \\
\hline
\end{tabular}

Tableau 4 : Matrice de transition entre 2005 et 2015

L'observation de la figure 5 montre globalement que les changements entre les catégories d'occupation des terres se sont opérés de façon différentielle sur le plan spatial dans le secteur d'étude. De ce fait, les savanes arborées et arbustives sont les unités qui ont le plus connu de pertes (67\%). Elles sont suivies par les forêts claires et les savanes boisées $(21 \%)$, catégories qui correspondent respectivement à une superficie de 338 055 ha et 106237 ha. Les champs et jachères ont enregistré le plus grand pourcentage de gain avec $75 \%$ des superficies, soit environ 386771 ha. La perte des unités forestières entraîne aussi celle de la biodiversité végétale dans le département de l'Alibori, et ce particulièrement dans les terroirs villageois fortement dégradés.

\subsection{Déforestation et dégradation forestière dans le département de l'Alibori}

La figure 6 présente le taux annuel de déforestation, de dégradation, d'amélioration et de reconstitution naturelle dans les aires protégées (AP), les terroirs villageois (TV) et dans l'ensemble du département de l'Alibori (ED).

L'analyse de la figure 6 montre qu'entre 2005 et 2015, le taux de déforestation sur l'ensemble du secteur est de $1,83 \%$ par an. Ce taux est de 3,10\% dans les terroirs villageois et de $0,70 \%$ dans les aires protégées (figure 6.1). La déforestation dans le secteur d'étude est issue de la conversion des forêts galeries, des forêts denses sèches, des forêts claires et savanes boisées et des savanes arborées et arbustives en mosaïques de champs et jachères. Les terroirs villageois sont plus touchés par la déforestation avec 4217,24 ha transformés en champs et jachères et en plantation chaque année.
Le taux de dégradation observé dans l'ensemble du département est de $0,30 \%$. Ce taux est légèrement plus élevé dans les aires protégées $(0,33 \%)$ que dans les terroirs villageois $(0,27 \%)$ (Figure 6.3). Néanmoins, les efforts de reboisement et de conservation semblent n'avoir qu'un faible impact sur le paysage, entraînant ainsi des taux annuels de reconstitution naturelle et d'amélioration du couvert végétal d'une valeur respective de $0,19 \%$ et de $0,04 \%$ dans le département de l'Alibori (Figures $6.2 ; 6.4$ ).

La figure 7 présente la proportion des terres touchées par la déforestation, la dégradation, l'amélioration et la reconstitution naturelle du paysage forestier dans le département de l'Alibori entre 2005 et 2015.

L'analyse de la figure 7 montre que les terroirs villageois sont plus touchés par la déforestation que les aires protégées, qui occupent près de $45 \%$ de la superficie du département. En effet, 14,96\% de la superficie totale du département sont touchés par la déforestation, plus de $20,45 \%$ des terroirs villageois et seulement $7,40 \%$ des aires protégées. Dans ces aires protégées, la forêt classée de l'Alibori supérieur est celle dans laquelle ce phénomène est le plus prononcé. Par ailleurs, 2,66 \% du département est touché par la dégradation des terres forestières, $1,40 \%$ a été reconstitué naturellement et seulement $0,35 \%$ a connu une amélioration.

Les photos 1,2 et 3 illustrent quelques pratiques qui entraînent la déforestation et la dégradation forestière dans le département de l'Alibori. 


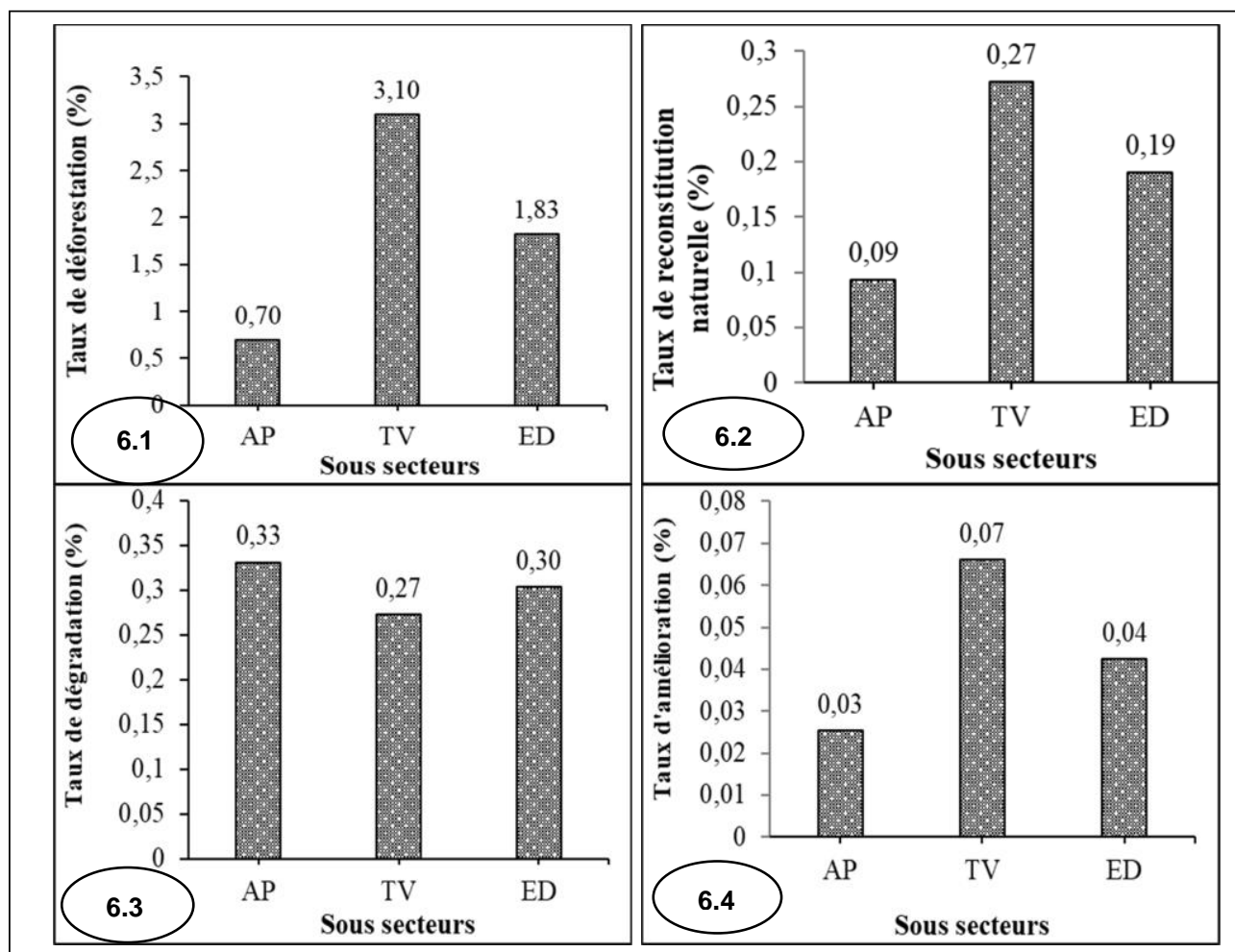

Figure 6 : Taux annuels de déforestation, de dégradation, d'amélioration et de reconstitution

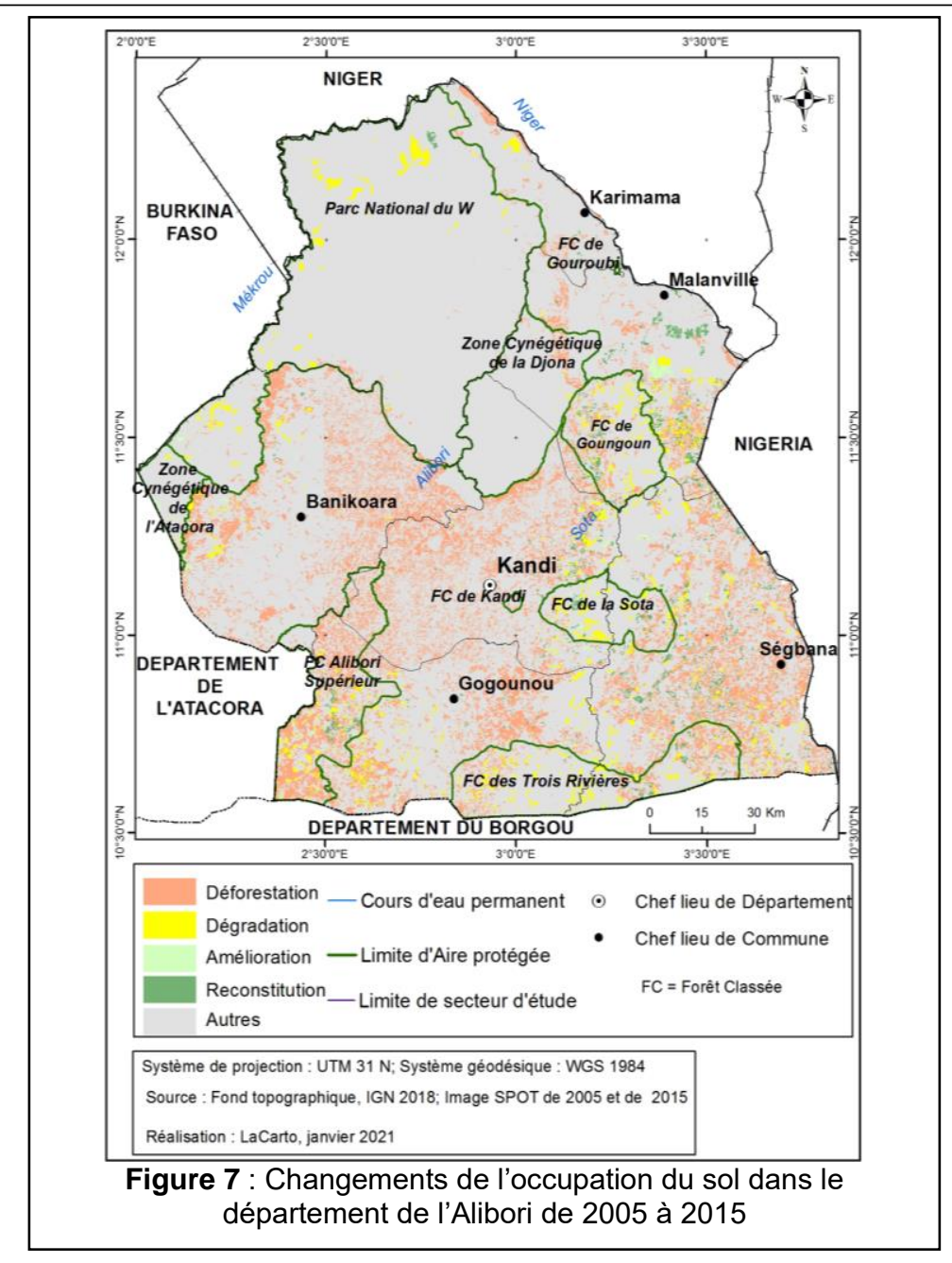

Revue Française de Photogrammétrie et Télédétection 
La photo 1 représente une étendue de sol dénudé dans la localité de Niambara, arrondissement de Lougou (commune de Ségbana).

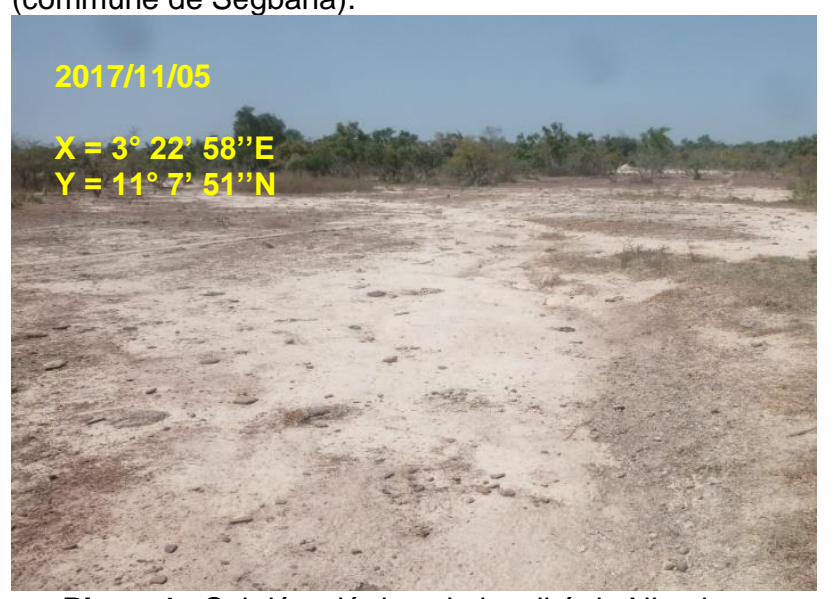

Photo 1 : Sol dénudé dans la localité de Niambara

Source : ISSIFOU MOUMOUNI Y., novembre 2017

On observe sur la photo 1 des sols dénudés abandonnés. Ces sols proviennent soit de la surexploitation agricole, soit du surpâturage et du package des troupeaux bovins. Ils peuvent naître également des feux de végétation ou des phénomènes naturels, comme l'assèchement (photo 1). Au cours du passage des troupeaux transhumants, les bouviers exercent l'émondage et l'étêtage des arbres fourragers, contribuant ainsi à la réduction de la biomasse aérienne ligneuse, et par ricochet, à la dégradation forestière.

La photo 2 illustre la pratique du feu de végétation à Tchakama dans la commune de Ségbana.

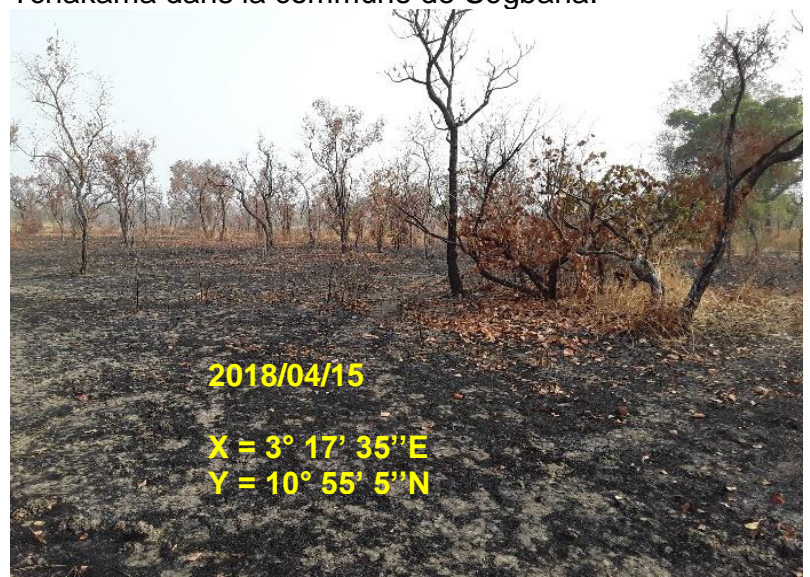

Photo 2 : Savane arbustive brulée à Tchakama

Source : ISSIFOU MOUMOUNI Y., janvier 2018

Les feux contribuent à la destruction du couvert végétal, surtout quand ils sont pratiqués de façon tardive.

On observe sur cette photo 2 la disparition quasi-totale de la strate herbacée après le passage des feux. La régénération est totalement calcinée, ainsi que le sousbois. Les feux répétitifs ralentissent la croissance des arbustes et finissent par avoir raison des plus vulnérables (arbres écorcés, déracinés, etc.). Un sol ainsi dénudé devient friable, plus vulnérables aux écoulements de surface et à l'érosion pluviale.

La photo 3 illustre la pratique du brûlis, mise en place à des fins d'augmentation des exploitations agricoles et surtout d'implantation de champs de coton dans la localité de Gbarana (commune de Ségbana).

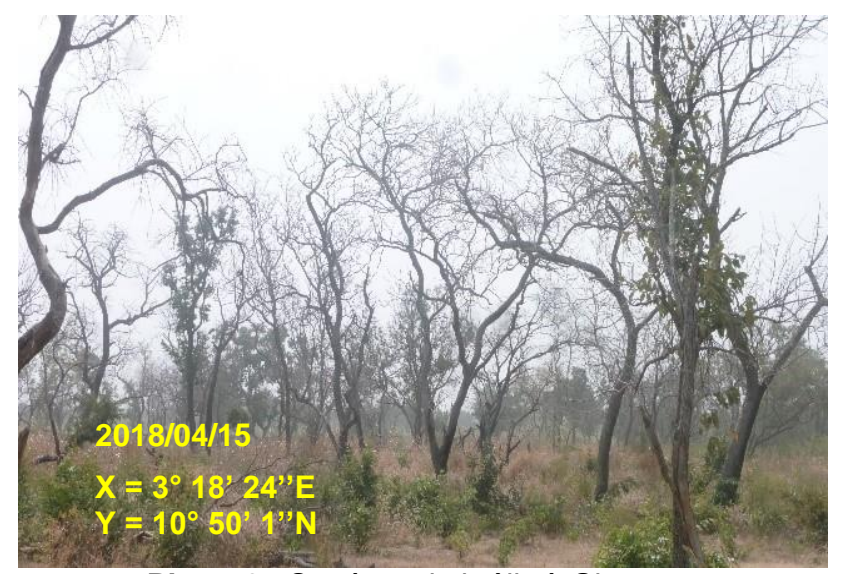

Photo 3 : Système de brûlis à Gbarana

Source : ISSIFOU MOUMOUNI Y., avril 2018

Sur la photo 3, on observe des arbres qui ont été brûlés dans le but de pouvoir installer des cultures dès la nouvelle saison de pluies. Des arbres sans distinction d'espèce sont brûlés sur pied pour permettre l'ensoleillement des cultures de coton. Ce carnage illustre parfaitement l'impact de l'expansion agricole sur la végétation naturelle, principal moteur de la déforestation dans le département de l'Alibori.

\section{Discussion}

Les résultats obtenus en appliquant la méthode basée sur la différence de classification, montrent que l'expansion des formations anthropiques a été réalisée au détriment des formations forestières naturelles entre 2005 et 2015. Cette méthode robuste de post-classification utilisée permet d'obtenir des résultats fiables [Coppin et al., 2004 ; Arouna, 2012].

Les forêts denses, les forêts claires et savanes boisées qui occupaient respectivement $0,12 \%$ et $8,06 \%$ du département en 2005 sont passées à $0,07 \%$ et 4,27 \% en 2015. Au cours de la période d'étude, $16,67 \%$ des forêts denses et $31,44 \%$ des forêts claires et savanes boisées se sont converties en savanes arborée et arbustive. Cette dégradation a provoqué une savanisation du milieu. Pour ce qui concerne l'anthropisation, ce sont les forêts denses $(20,83 \%)$, les forêts galeries $(11,22 \%)$, les forêts claires et savanes boisées $(19,22 \%)$, les forêts et savanes marécageuses $(31,50 \%)$ ainsi que les savanes arborée et arbustive $(20,62 \%)$ qui ont été converties en mosaïques de cultures et jachères. Ces dernières ont aussi cédé une partie de leur superficie aux habitats humains (0,05\%). Cette anthropisation a entrainé une forte déforestation dans le milieu. L'hypothèse qui stipule que le département de l'Alibori a enregistré une déforestation, entre 2005 et 2015, aussi bien dans les terroirs villageois que les dans les aires protégées, est alors vérifiée. 
Zakari (2015) a constaté la régression de toutes les formations végétales naturelles dans le bassin versant de la Sota. Cette régression est plus marquée par la régression des forêts claires $\left(196,76 \mathrm{~km}^{2} / \mathrm{an}\right)$, des galeries forestières $\left(196,76 \mathrm{~km}^{2} / \mathrm{an}\right)$ et des forêts denses $(1,98$ $\left.\mathrm{km}^{2} / \mathrm{an}\right)$. Elle est réalisée au profit des champs et jachères, qui ont connu une expansion moyenne annuelle de 113,47 km² entre 1995 et 2013.

Dans le bassin cotonnier-est du Bénin, Toko Imorou et al. (2019) ont observé la régression des forêts claires $(505,18$ $\left.\mathrm{km}^{2}\right)$, des galeries forestières $\left(271,22 \mathrm{~km}^{2}\right)$ et des forêts denses sèches $\left(54,99 \mathrm{~km}^{2}\right)$. Le constat de la disparition du couvert forestier est un fait. En effet, les études récentes font état d'un recul du couvert forestier national, qui est passé de 31,6\% en 1990 à 30,6 \% en 2015 [FAO, 2018 ; Biaou et al., 2019]. Les conclusions de ces travaux corroborent les résultats de la présente recherche.

Entre 2005 et 2015, le taux de déforestation sur l'ensemble du département de l'Alibori est de 1,83\% par an. Ce taux est de 3,10\% dans les terroirs villageois et de 0,70\% dans les aires protégées. Le taux de dégradation observé dans l'ensemble du département est de 0,30\%. Ce taux est légèrement plus élevé dans les aires protégées $(0,33 \%)$ que dans les terroirs villageois $(0,27 \%)$. Les efforts de reboisement sont notés mais sont insuffisants pour compenser la déforestation et la dégradation des forêts enregistrées. En effet, les taux annuels de reconstitution naturelle et d'amélioration du couvert végétal sont respectivement de $0,19 \%$ et de $0,04 \%$ dans le département de l'Alibori. Ces résultats sont inférieurs à ceux obtenus par plusieurs auteurs. Dans l'ensemble du bassin moyen de la Sota, le taux annuel de déforestation nette est de 2,03 \% (soit 2 669,89 ha/an). Cette déforestation est évaluée à 465,46 ha/an (soit 1,01\%) dans les aires protégées, alors qu'elle s'élève à 2 204,74 ha/an (soit 2,59 \%/an) dans les terroirs [Issifou Moumouni, 2019]. De plus, selon Kouta (2020), la proportion de la superficie du paysage forestier dans le bassin cotonnier du nord-Bénin a connu une régression de 1986 à 2000, soit une variation de $13,78 \%$. Cela correspond à une superficie de 43101,30 ha de moins qu'en 1986. Cette régression devient plus rapide entre 2000 et 2016, période au cours de laquelle la variation a été de $36,03 \%$, soit 2,52 fois supérieure à celle observée entre 1986 et 2000. Cela représente une transformation de 112715,50 ha du paysage forestier du bassin.

Les principaux moteurs de la déforestation enregistrée dans le département de l'Alibori sont l'agriculture, l'élevage extensif et l'exploitation forestière sous l'impulsion de la croissance démographique.

La population du département de l'Alibori est passée de 521093 habitants en 2002 [INSAE, 2004] à 867463 habitants en 2013 [INSAE, 2016]. Cette croissance démographique, facteur indirect important de la déforestation et de la dégradation des forêts, aura des conséquences pour les ressources naturelles.

Au Bénin comme dans la plupart des pays africains, la plupart des politiques agricoles visent l'augmentation de la production par l'accroissement des superficies emblavées
[Roper, 2019]. Ainsi, pour accroître la production agricole, les agriculteurs sont obligés d'étendre les superficies cultivées, ce qui se traduit par des défrichements. Selon Domingo (2007), les centres d'action régionaux pour le développement rural (CARDER) au Bénin ont adopté une approche productiviste qui, à coup de volumes de production et de prescriptions de quota, ont accéléré le déboisement.

L'essentiel du fourrage consommé par les bovins en saison sèche dans le département de l'Alibori provient essentiellement des espèces fourragères ligneuses. L'exploitation forestière frauduleuse est aussi pratiquée dans ce département. Le code forestier constitué de la loi n 93-009 du 2 juillet 1993 portant régime des forêts en République du Bénin [RB, 1993] et de son décret d'application $n^{\circ}$ 96-271 du 2 juillet 1996 [RB, 1966], est antérieur à la décentralisation et ne donne pratiquement pas de compétences aux conseils communaux, surtout en matière de gestion des aires protégées. Cette désuétude du code forestier contribue indirectement à la déforestation et à la dégradation des forêts.

Ainsi, l'agriculture, l'élevage extensif et l'exploitation forestière contribuent directement à la déforestation et à la dégradation du couvert forestier dans le département de l'Alibori [Arouna et al., 2016 ; Issifou Moumouni, 2020 ; Kouta, 2020]. Ces facteurs directs sont sous-tendus par des facteurs indirects, comme ceux de la croissance démographique, des politiques agricoles et du code forestier.

\section{Conclusion}

La présente recherche portant sur la cartographie de la déforestation à base de l'imagerie satellitaire dans le département de l'Alibori au Nord du Bénin, a permis de mettre en évidence l'importance des outils de télédétection pour la compréhension des changements spatio-temporels de l'occupation des terres et pour le suivi des formations végétales naturelles. La cartographie opérée à partir des images satellites SPOT 5 et 7 de 2005 et 2015 a montré qu'il y a une forte régression de toutes les formations forestières au profit des formations anthropiques. Cette régression est provoquée par la forte dégradation des formations végétales naturelles, marquée par une réduction des forêts galeries, des forêts denses, des forêts claires et savanes boisées et des savanes arborées et arbustives. Cette dégradation a entraîné une déforestation poussée, marquée par l'extension galopante des champs et jachères, des plantations et des habitats humains dans les aires protégées comme dans les terroirs villageois. Cette dégradation est plus prononcée dans les aires protégées que dans les terroirs, alors que la déforestation est plus élevée dans les terroirs que dans les aires protégées. Néanmoins on note une amélioration et une reconstitution naturelles des formations végétales naturelles en cours dans certains secteurs. Celles-ci sont plus importantes dans les terroirs que dans les aires protégées, du fait des efforts de reboisements opérés dans le département de l'Alibori par des acteurs privés et publics. Toutefois, cette reconstitution naturelle est loin de compenser la déforestation et la dégradation enregistrées au cours de la période 2005-2015. La politique agricole

Revue Française de Photogrammétrie et Télédétection 
constitue l'un des facteurs importants de cette transformation rapide du paysage du département de l'Alibori.

II importe donc de trouver un modèle de développement local durable intégrant les bonnes pratiques en matière d'affectation des terres, et respectueux de l'environnement.

\section{Références}

Afaq Y., Manocha A., 2021. Analysis on change detection techniques for remote sensing applications: A review. Ecological Informatics, Volume 63. https://doi.org/10.1016/j.ecoinf.2021.101310.

Arouna O., 2012. Cartographie et modélisation prédictive des changements spatio-temporels de la végétation dans la Commune de Djidja au Bénin : Implication pour l'aménagement du territoire. Thèse de doctorat en Géographie, UAC, 246 p.

Arouna O., Etene C. G., Issiako D., 2016. Dynamique de l'occupation des terres et état de la flore et de la végétation dans le bassin supérieur de l'Alibori au Benin. J. Appl. Biosci, 108 : 10543-10552.

Biaou S., Houeto F, Gouwakinnou G, Biaou S. H., Awessou B., Tovihessi S., Tete R., 2019. Dynamique spatio-temporelle de l'occupation du sol de la forêt classée de Ouénou-Bénou au Nord Bénin. Conférence OSFACO : Des images satellites pour la gestion durable des territoires en Afrique, Mar 2019, Cotonou, Bénin. Hal-02189367.

Blanc P., 1999. Développement de méthodes pour la détection de changement. Thèse de Doctorat, Sciences de la Terre, École Nationale Supérieure des Mines de Paris, $205 \mathrm{p}$.

Bogaert, J., Barima, Y. S., lyongo, W. M. L., Bamba, I., Mama, A., Toyi, M., \& Lafortezza, R., 2011. Forest fragmentation: causes, ecological impacts and implications for landscape management. Landscape Ecology in Forest Management and Conservation, 273-296.

Coppin P., Jonckheere I., Nackaerts I., Muys B., Lambin E., 2004. Review Article Digital change detection methods in ecosystem monitoring: a review. International Journal of Remote Sensing, 25(9):1565-1596.

De Wasseige C., Devers D., de Marcken P., Ebaa Atyi R., Robert N., Mayaux P., 2008. The Forests of the Congo Basin: State of the Forest 2008. Luxembourg : Office of the European Union.

Domingo E., 2007. La région urbaine du littoral au Bénin : dynamiques urbanisantes et environnement, une géographie de l'aménagement du territoire. Thèse de doctorat d'Etat, (Tomes 1 et 2), Université de Lomé, 588 p.

Egah J, Baco M. N., Lokossou R., Moutouama F. T., Akponikpè P., Fatondji D., Djènontin J., Tossou C. R., Sokpon N., 2014. " Incidence économique des techniques exogènes de conservation de l'eau et des sols au Bénin ", Bulletin de la Recherche Agronomique du Bénin (BRAB) Numéro 75 - Volume 1 - Juin 2014 BRAB en ligne (on line) sur le site web http://www.slire.net ISSN sur papier (on hard copy) : 1025-2355 et ISSN en ligne (on line) : 18407099

FAO, 2018. La situation des forêts du monde. Les forêts au service du développement durable, Licence : CC BYNC-SA 3.0 IGO, p 158.

FAO, 2017. Monitoring deforestation and forest degradation. FAO Regional Office for Latin America and the Caribbean. http://www.fao.org/3/a-bt193e.pdf.

$\mathrm{FAO}$, 2016. Considérations techniques relatives à l'établissement de niveaux d'émissions de référence pour les forêts et/ou niveaux de référence pour les forêts dans le contexte de la REDD+ au titre de la CCNUCC. Programme ONU-REDD, Italie (disponible à http://www.unredd.net/index.php?option=com_docman\&t ask=doc_download\&gid=13469\&ltemid=53)

Fournier A., 2008. Détection et classification de changements sur des scènes urbaines en télédétection. Thèse de doctorat, Institut Supérieur de l'Aéronautique et de l'Espace, Université de Toulouse, 129 p.

Hasan A. F., 2019. Évaluation de la dégradation des forêts primaires par télédétection dans un espace de front pionnier consolidé d'Amazonie orientale (Paragominas), Thèse de doctorat en géographie physique (Le Mans université), $232 \mathrm{p}$.

Hountondji Y., Gaoue O. G., Sokpon N., Ozer P., 2013. Analyse éco géographique de la fragmentation du couvert végétal au nord-Bénin : paramètres dendrométriques et phytoécologiques comme indicateurs in situ de la dégradation des peuplements ligneux. Geo-Eco-Trop., 37 (1) : 53-70.

IFN, 2007. Rapport d'exécution de l'Inventaire Forestier National (IFN), Projet Bois de Feu, phase II. Cotonou, Bénin, Ministère de l'Environnement et de la Protection de la Nature, $41 \mathrm{p}$.

INSAE, 2004. Cahier des villages et quartiers de ville du département de l'Alibori. Cotonou, Bénin, 36 p.

INSAE, 2010. Enquête Modulaire Intégrée sur les Conditions de Vie des Ménages, Cotonou, Bénin, 86 p.

INSAE, 2016. Cahier des villages et quartiers de ville du département de l'Alibori. Cotonou, Bénin, 26 p.

Issifou Moumouni Y., 2020. Evaluation de la Déforestation et de la Dégradation du couvert végétal et estimation des Gaz à Effet de Serre correspondants dans le Bassin moyen de la Sota au Nord-Bénin, Thèse de Doctorat, Université d'Abomey-Calavi, Bénin, 279 p.

Lu D., Mausel P., Brondizio E. \& Moran E., 2004. Change detection techniques. International Journal of Remote Sensing, 25(12): 2365-2401.

Kioko J., Okello M. M., 2010. Land use cover and environmental changes in a semi-arid rangeland, Southern Kenya. J. Geogr. Regional Plann. 3(11):322-326.

Kouta S., 2020. Modélisation de la dynamique de l'occupation des terres et résilience de la flore dans le bassin cotonnier du Nord-Benin. Thèse de Doctorat, Université d'Abomey-Calavi, Bénin, 278 p. 
Kouta S., Toko Imorou I., Sogbosi E, Mazo I., Zakari S., Arouna O., Djaouga M., Thomas O., 2018. Classification d'images satellites SPOT avec randomforest et cartographie du paysage forestier dans le bassin cotonnier du Nord-Benin. In Actes de la conférence scientifique internationale OSFACO : Images satellitaires pour un meilleur aménagement des territoires et une gestion durable de la biodiversité, p. 211-228

Mama A., Oumorou M., Sinsin B., De Canniere C., Bogaert J., 2020. Anthropisation des paysages naturels des aires protégées au Bénin : Cas de la Forêt Classée de l'Alibori Supérieur (FC-AS). American Journal of Innovative Research and Applied Sciences, 11(2):117-125.

Mama A., Sinsin B., De Canniere C., Bogaert J., 2013. Anthropisation et dynamique des paysages en zone soudanienne au nord du Bénin. Tropicultura, 31(1): 78-88.

Maman, S., Orlovsky, L., Blumberg, D. G., Berliner, P., \& Mamedov, B., 2011. A landcover change study of takyr surfaces in Turkmenistan. Journal of arid environments, 75(9), 842-850.

Olaniyi A. A. Ajadi , Franz J. M., Webley W. P., 2016. Change Detection in Synthetic Aperture Radar Images Using a Multiscale-Driven Approach. Remote Sensing, 8, 482, doi:10.3390/rs8060482.

Oloukoi J., Mama V., Agbo F. B., 2006. Modélisation de la dynamique de l'occupation des terres dans le département des Collines au Bénin. Télédétection, 6 (4) : 305-323.

Ortega M. X., Bermude J. D. Z., Happ P. N., Gomes A., Feitosa R. Q., 2019. Evaluation of deep learning techniques for deforestation detection in the amazon forest. ISPRS Annals of the Photogrammetry, Remote Sensing and Spatial Information Sciences, Volume IV2/W7, PIA19+MRSS19 - Photogrammetric Image Analysis \& Munich Remote Sensing Symposium, 18-20 September 2019, Munich, Germany

Provencher-Nolet L., 2014. Détection de changement à court terme de la toundra arbustive à partir de photographies aériennes, région d'Umiujaq, Nunavik (Québec, Canada). Mémoire de Master, Université du Québec, 150 p.

RB (République du Bénin), 1996. Décret n 96-271 du 2 juillet 1996 portant modalités d'application de la Loi $n^{\circ} 93-$ 009 du 2 juillet 1993 portant régime des forêts en République du Bénin. DFRN, Cotonou, Bénin, 24 p.

RB (République du Bénin), 1993. Loi n 93-009 du 2 juillet 1993 portant régime des forêts en République du Bénin. DFRN, Cotonou, Bénin, 26 p.

Roper J., 1999. Déforestation : le déclin des forêts tropicales. ACDI, Québec, Canada. http://www.rcfacfan.org/french/f.issues.12.html

Scouvart M., Lambin E. F, 2006. Approche systémique des causes de la déforestation en Amazonie brésilienne : syndromes, synergies et rétroactions. L'Espace Géographique, 3 : 241-254.

Tchatchou B., Sonwa D., Ifo A.S., Tiani A. M., 2015. Déforestation et dégradation des forêts dans le Bassin du
Congo : État des lieux, causes actuelles et perspectives. Papier occasionnel 120. Bogor, Indonesie : CIFOR, 60 p.

Toko Imorou I., Arouna O., Zakari S., Djaouga M., Thomas O., Kinmadon G., 2019. Évaluation de la déforestation et de la dégradation des forêts dans les aires protégées et terroirs villageois du bassin cotonnier du Bénin, 2019. Conférence OSFACO : Des images satellites pour la gestion durable des territoires en Afrique, Mars 2019, Cotonou, Bénin, 25 p. 〈hal-02189556〉.

Toko Mouhamadou I., 2014. Facteurs déterminants de la fragmentation des écosystèmes forestiers : cas des îlots de forêts denses sèches de la forêt classée des Monts Kouffé et de sa périphérie au Bénin, Université d'AbomeyCalavi, Bénin, 203 p.

Vissin E. W., 2007. Impact de la variabilité climatique et de la dynamique des états de surface sur les écoulements du bassin béninois du Niger. Thèse de doctorat. Université de Bourgogne, Dijon, France, 311 p.

Zakari S., 2015. Vulnérabilité des parcours naturels aux changements climatiques dans le bassin de la Sota à l'exutoire de Couberi (Bénin). Thèse de Doctorat, Université d'Abomey-Calavi, Bénin, 203 p.

\section{TITRE EN ANGLAIS}

\section{MAPPING DEFORESTATION IN THE DEPARTMENT OF ALIBORI (NORTHERN BENIN) USING SATELLITE IMAGERY SPOT}

\section{LEGENDES EN ANGLAIS}

\section{List of Figures}

Figure 1: Location of the department of Alibori

Figure 2: Color composite and some ground control points Figure 3: State of land cover and land use in the department of Alibori in 2005 and 2015

Figure 4: Overall rate of change in natural forests, plantations, and other non-forested formations between 2005 and 2015

Figure 5: Gains and losses of land cover and land use from 2005 to 2015

Figure 6: Annual rates of deforestation, degradation, improvement, and restoration

Figure 7: Changes in land cover and land use in the department of Alibori from 2005 to 2015

\section{List of Tables}

Table 1: Characteristics of the images covering the study area

Table 2: Deforestation and degradation analysis matrix

Table 3: Confusion matrix

Table 4: Transition matrix between 2005 and 2015

List of Photographs

Photograph 1: Bare soil in the locality of Niambara

Photograph 2: Burnt shrubby savanna in Tchakama

Photograph 3: Slash-and-burn system in Gbarana 